\title{
POSITIVE SCALAR CURVATURE AND AN EQUIVARIANT CALLIAS-TYPE INDEX THEOREM FOR PROPER ACTIONS
}

\author{
HAO GUO, PETER HOCHS, AND VARGHESE MATHAI
}

\begin{abstract}
For a proper action by a locally compact group $G$ on a manifold $M$ with a $G$-equivariant Spin-structure, we obtain obstructions to the existence of complete $G$-invariant Riemannian metrics with uniformly positive scalar curvature. We focus on the case where $M / G$ is noncompact. The obstructions follow from a Callias-type index theorem, and relate to positive scalar curvature near hypersurfaces in $M$. We also deduce some other applications of this index theorem. If $G$ is a connected Lie group, then the obstructions to positive scalar curvature vanish under a mild assumption on the action. In that case, we generalise a construction by Lawson and Yau to obtain complete $G$-invariant Riemannian metrics with uniformly positive scalar curvature, under an equivariant bounded geometry assumption.
\end{abstract}

\section{Contents}

1. Introduction

Results on positive scalar curvature 2

A Callias-type index theorem 4

Outline of this paper

Acknowledgements

Notation and conventions 5

2. Results on positive scalar curvature 6

2.1. Obstructions 6

2.2. Existence for connected Lie groups 8

3. An index theorem 9

3.1. The $G$-Callias-type index 9

3.2. Hypersurfaces and the index theorem 11

4. Properties of the $G$-Callias-type index 12

4.1. Sobolev modules 12

4.2. Vanishing 15

4.3. Homotopy invariance 16

4.4. A relative index theorem 16

5. Proof of the $G$-Callias-type index theorem 18

5.1. An index on the cylinder 19

5.2. Attaching a half-cylinder 20

5.3. Proof of Proposition 5.1 22 
5.4. Proof of Theorem 3.4 25

6. Proofs of results on positive scalar curvature 27

6.1. Obstruction results 27

6.2. Existence result 28

7. Further applications of the Callias-type index theorem 30

7.1. The image of the assembly map 30

7.2. Cobordism invariance of the assembly map 31

7.3. Spin ${ }^{c}$-Dirac operators 31

7.4. Induction 32

7.5. Callias quantisation commutes with reduction 33

References 34

\section{INTRODUCTION}

Let $G$ be a locally compact group, acting properly on a manifold $M$. Suppose that $M$ has a $G$-equivariant Spin-structure. The results in this paper are about the following question.

Question 1.1. When does $M$ admit a complete, G-invariant Riemannian metric with uniformly positive scalar curvature?

We are mainly interested in the case where $M / G$ is noncompact.

The literature on the non-equivariant case of Question 1.1 is too vast to summarise, but important work where $M$ is noncompact was done by Gromov and Lawson [13. A more refined perspective on the non-equivariant case is to consider a manifold $X$, and let $M$ be its universal cover, and $G$ is fundamental group. This allows one to construct obstructions to metrics of positive scalar curvature in terms of $G$-equivariant index theory on $M$, refining index-theoretic obstructions on $X$. If $X$ is compact, this is the origin of the even more refined Rosenberg index [36, 35, 37, in $K O$-theory of the real maximal group $C^{*}$-algebra of $G$.

More generally, if $G$ is a discrete group not necessarily acting freely on $M$, then $X:=M / G$ is an orbifold, whence Question 1.1 becomes the question of whether $X$ admits an orbifold metric of positive scalar curvature.

We consider the case where $G$ is not necessarily discrete, and does not necessarily act freely. Results on this case of Question 1.1 in the case where $G$ is an almost-connected Lie group and $M / G$ is compact were obtained in [18, 34]. In this paper, we focus on the case where $M / G$ is noncompact.

Results on positive scalar curvature. We first obtain obstructions to $G$-invariant Riemannian metrics with positive scalar curvature, both in the $K$-theory of the maximal or reduced group $C^{*}$-algebra of $G$, and in terms of numerical topological invariants generalising the $\hat{A}$-genus. If $G$ is a connected Lie group, then these obstructions vanish under a mild assumption, 
as shown in [21]. In that case, we construct $G$-invariant Riemannian metrics with uniformly positive scalar curvature, under an equivariant bounded geometry assumption.

Our most general obstruction result is the following.

Theorem 1.2. Let $H \subset M$ be a $G$-invariant, cocompact hypersurface with trivial normal bundle, that partitions $M$ into two open sets. If $M$ admits a complete, G-invariant Riemannian metric with nonnegative scalar curvature, and positive scalar curvature in a neighbourhood of $H$, then

$$
\operatorname{index}_{G}\left(D^{H}\right)=0 \quad \in K_{*}\left(C^{*}(G)\right),
$$

for a Spin-Dirac operator $D^{H}$ on $H$.

See Theorem 2.1. In the case where $M$ is the universal cover of a manifold $X$ and $G$ is its fundamental group, this becomes Theorem A in [9].

Theorem 1.2 implies topological obstructions to $G$-invariant Riemannian metrics with positive scalar curvature. Let $g \in G$, and let $H^{g} \subset H$ be the fixed point set of $g$. Let $\mathcal{N} \rightarrow H^{g}$ be its normal bundle in $H$, and let $R^{\mathcal{N}}$ be the curvature of the Levi-Civita connection restricted to $\mathcal{N}$. The $g$-localised $\hat{A}$-genus of $H$ is

$$
\hat{A}_{g}(H):=\int_{H^{g}} c^{g} \frac{\hat{A}\left(H^{g}\right)}{\operatorname{det}\left(1-g e^{-R^{\mathcal{N}} / 2 \pi i}\right)^{1 / 2}},
$$

for a cutoff function $c^{g}$ on $X^{g}$. If $G$ acts freely, then $\hat{A}_{g}(H)=0$ if $g \neq e$, and $\hat{A}_{e}(H)$ is the $\hat{A}$-genus of $H / G$. In general, for example in the orbifold case, $\hat{A}_{g}(H)$ may be nonzero for different $g$.

Corollary 1.3. Consider the setting of Theorem 1.2. Suppose that either

- $G$ is any locally compact group and $g=e$;

- $G$ is discrete and finitely generated and $g$ is any element; or

- $G$ is a connected semisimple Lie group and $g$ is a semisimple element.

Then $\hat{A}_{g}(H)$ is independent of the choice of G-invariant Riemannian metric, and $\hat{A}_{g}(H)=0$.

See Theorem 2.4.

Theorem 2 in [21] is a generalisation of Atiyah and Hirzebruch's vanishing result [3] to the noncompact case. It states that, if $G$ is connected, and not every stabiliser of its action on $H$ is maximal compact, then $\operatorname{index}_{G}\left(D^{H}\right)=$ 0 . This implies that $\hat{A}_{g}(H)=0$ as well. In view of Theorem 1.2 and Corollary 1.3 , this makes it a natural question if $M$ admits a $G$-invariant Riemannian metric with positive scalar curvature if $G$ is a connected Lie group. The answer, given in the present paper, turns out to be yes under a certain equivariant bounded geometry assumption.

Suppose that $G$ is a connected Lie group, and let $K<G$ be a maximal compact subgroup. Abels' slice theorem [1 implies that there is a diffeomorphism $M \cong G \times{ }_{K} N$, for a $K$-invariant submanifold $N \subset M$. Consider 
the infinitesimal action map

$$
\varphi: N \times \mathfrak{k} \rightarrow T N
$$

mapping $(y, X) \in N \times \mathfrak{k}$ to $\left.\frac{d}{d t}\right|_{t=0} \exp (t X) y$. The action by $K$ on $N$ is said to have no shrinking orbits with respect to a $K$-invariant Riemannian metric on $N$, if the pointwise operator norm of $\varphi$ as a map from $\mathfrak{k}$ to a tangent space is uniformly positive outside a neighbourhood of the fixed point set $N^{K}$. We say that $N$ has $K$-bounded geometry if it has bounded geometry and no shrinking orbits.

Theorem 1.4. Suppose that $K$ is non-abelian, and that $K$ acts effectively on $N$ with compact fixed point set. If there exists a $K$-invariant Riemannian metric on $N$ for which $N$ has $K$-bounded geometry, then the manifold $G \times{ }_{K} N$ admits a $G$-invariant metric with uniformly positive scalar curvature.

In the compact case, the Atiyah-Hirzebruch vanishing theorem [3] implies that the obstruction $\hat{A}(N)$ to Riemannian metrics of positive scalar curvature vanishes if $K$ acts nontrivially on $N$. Then Lawson and Yau [27] constructed such metrics, under mild conditions. In a similar way, Theorem 1.4 complements the vanishing result in [21].

A Callias-type index theorem. Two effective sources of index-theoretic obstructions to metrics of positive scalar curvature on noncompact manifolds are coarse index theory and Callias-type index theory. For some results involving coarse index theory, see for example [38] and the literature on the coarse Novikov conjecture, in particular [12 for the equivariant setting we are interested in here. We will use Callias-type index theory.

Not assuming that $M$ is Spin for now, and letting $G$ be any locally compact group, we consider a $G$-equivariant Dirac-type operator $D$ on a $G$ equivariant vector bundle $S \rightarrow M$. A Callias-type operator is of the form $D+\Phi$, for a $G$-equivariant endomorphism $\Phi$ of $S$ such that $D+\Phi$ is uniformly positive outside a cocompact set. Then this operator has an index index $_{G}(D+\Phi) \in K_{*}\left(C^{*}(G)\right)$, constructed in [15]. (See Theorem 4.2 in [17] for a realisation of this index in terms of coarse geometry.)

Theorem 1.5 (G-Callias-type index theorem). We have

$$
\operatorname{index}_{G}(D+\Phi)=\operatorname{index}_{G}\left(D_{N}\right) \text {, }
$$

for a Dirac operator $D_{N}$ on a $G$-invariant, cocompact hypersurface $N \subset M$.

See Theorem 3.4. Versions of this result where $G$ is trivial were proved in 2, 5, 7, 8, 25. Versions for operators on bundles of modules over operator algebras were proved in [6, 9]. Parts of our proof of Theorem 1.5 are based on a similar strategy as the proof of the index theorem in $[9$.

We deduce Theorem 2.1 from Theorem 1.5. This approach is an equivariant generalisation of the obstructions to metrics of positive scalar obtained in $[2,7,9]$. 
If $g \in G$, then under conditions, there is a subalgebra $A(G) \subset C^{*}(G)$ such that $K_{*}(A(G))=K_{*}\left(C^{*}(G)\right)$, and there is a well-defined trace $\tau_{g}$ on $A(G)$ given by

$$
\tau_{g}(f)=\int_{G / Z} f\left(h g h^{-1}\right) d(h Z),
$$

where $Z$ is the centraliser of $g$. In various settings, including the three cases in Corollary 1.3, there are index formulas for the number $\tau_{g}\left(\operatorname{index}_{G}(D)\right)$, see [24, 41, 40]. These index formulas imply that, in the setting of Theorem 1.2 .

$$
\tau_{g}\left(\operatorname{index}_{G}\left(D^{H}\right)\right)=\hat{A}_{g}(H) .
$$

Hence Theorem 1.2 implies Corollary 1.3 .

Theorem 1.4 is proved via a generalisation of Lawson and Yau's arguments [27, together with a result from [17] that allows one to induce up metrics of positive scalar curvature from $N$ to $M=G \times_{K} N$.

Apart from using Theorem 1.5 to prove Theorem 2.1, we obtain some further applications, on the image (Corollary 7.1) and cobordism invariance (Corollary 7.2) of the analytic assembly map; on the Callias-type index of Spin $^{c}$-Dirac operators (Corollary 7.3); on induction of Callias-type indices from compact groups to noncompact groups (Corollary 7.4); and on the Spin $^{c}$-version [31] of the quantisation commutes with reduction problem [14, 28, 30, 39] for Spin ${ }^{c}$-Callias type operators (Corollary 7.5).

Outline of this paper. We state our obstruction and existence results in Section 2, Theorem 2.1. Corollary 2.4 and Theorem 2.9. In Section 3, we state the equivariant Callias-type index theorem, Theorem 3.4. Theorem 3.4 is proved in Sections 4 and 5 . We then deduce Theorem 2.1 and Corollary 2.4 in Subsection 6.1. Theorem 2.9 is proved in Subsection 6.2. In Section 7. we obtain some further applications of the Callias-type index theorem, Corollaries 7.17 .5 .

Acknowledgements. HG was supported in part by funding from the National Science Foundation under grant no. 1564398. PH thanks Guoliang Yu and Texas A\&M University for their hospitality during a research visit. VM was supported by funding from the Australian Research Council, through the Australian Laureate Fellowship FL170100020.

Notation and conventions. All manifolds, vector bundles, group actions and other maps between manifolds are implicitly assumed to be smooth. If a Hilbert space $H$ is mentioned, the inner product on that space will be denoted by $(-,-)_{H}$, and the corresponding norm by $\|\cdot\|_{H}$. Spaces of continuous sections are denoted by $\Gamma$; spaces of smooth sections by $\Gamma^{\infty}$. Subscripts $c$ denote compact supports.

If $G$ is a group, and $H$ is a subgroup acting on a set $S$, then we write $G \times{ }_{H} S$ for the quotient of $G \times S$ by the $H$-action given by

$$
h \cdot(g, s)=\left(g h^{-1}, h s\right),
$$


for $h \in G, g \in G$ and $s \in S$. If $S$ is a manifold, $G$ is Lie group and $H$ is a closed subgroup, then this action is proper and free, and $G \times_{H} S$ is a manifold.

A continuous group action, and also the space acted on, is said to be cocompact if the quotient space is compact.

\section{Results on POSitive SCALAR CURVATURE}

In all parts of this paper except Subsections 2.2 and 6.2, which concern the existence results, $G$ will be be a locally group, acting properly on a manifold $M$. We do not assume that $M / G$ is compact and are in fact interested mainly in the case where it is not. The group $G$ may have infinitely many connected components, and for may for example be an infinite discrete group.

2.1. Obstructions. For a proper, cocompact action by $G$ on a manifold $N$, a $G$-equivariant elliptic differential operator $D$ on $N$ has an equivariant index $\operatorname{index}_{G}(D) \in K_{*}\left(C^{*}(G)\right)$ defined by the analytic assembly map [4]. Here $C^{*}(G)$ is the maximal or reduced group $C^{*}$-algebra of $G$, and the index takes values in its even $K$-theory if $D$ is odd with respect to a grading, and in odd $K$-theory otherwise.

Our most general obstruction result is the following.

Theorem 2.1. Let $M$ be a complete Riemannian Spin-manifold, on which a locally compact group $G$ acts properly and isometrically. Let $H \subset M$ be a $G$-invariant, cocompact hypersurface with trivial normal bundle, such that $M \backslash H=X \cup Y$ for disjoint open subsets $X, Y \subset M$. If the scalar curvature on $M$ is nonnegative, and positive in a neighbourhood of $H$, then the SpinDirac operator $D^{H}$ on $H$, acting on sections of the restriction of the spinor bundle on $M$ to $H$, satisfies

$$
\operatorname{index}_{G}\left(D^{H}\right)=0 \quad \in K_{*}\left(C^{*}(G)\right) .
$$

We will deduce this result from an equivariant index theorem for Calliastype operators, Theorem 3.4, which may be of independent interest and has some other applications as well.

Remark 2.2. If $M$ is the universal cover of a manifold $X$, and $G$ is the fundamental group of $X$ acting on $M$ in the usual way, then Theorem 2.1 reduces to Theorem A in [9], by the Miščenko-Fomenko realisation of the analytic assembly map in that case [29].

Theorem 2.1 implies a set of topological obstructions to $G$-invariant positive scalar curvature metrics on $M$. Let $X$ be any Riemannian manifold on which $G$ acts properly, isometrically and cocompactly. Let $g \in G$, and let $X^{g} \subset X$ be its fixed point set. (Properness of the action implies that $X^{g}=\emptyset$ if $g$ is not contained in a compact subset of $G$.) Let $\mathcal{N} \rightarrow X^{g}$ be the normal bundle to $X^{g}$ in $X$. The connected components of $X^{g}$ are submanifolds of $X$ of possibly different dimensions, so the rank of $\mathcal{N}$ may jump between 
these components. In what follows, we implicitly apply all constructions to the connected components of $X^{g}$ and add the results together.

By a cutoff function we will mean a smooth function $c: M \rightarrow[0,1]$ such that $\operatorname{supp}(c)$ has compact intersection with each $G$-orbit, and for each $x \in M$ we have

$$
\int_{G} c\left(g^{-1} x\right) d g=1
$$

We will also use cutoff functions for other group actions, which are defined analogously.

Let $R^{\mathcal{N}}$ be the curvature of the Levi-Civita connection restricted to $\mathcal{N}$. Let $\hat{A}\left(X^{g}\right)$ be the $\hat{A}$-class of $X^{g}$. Let $Z_{G}(g)<G$ be the centraliser of $g$. Let $c^{g}$ be a cutoff function for the action by $Z_{G}(g)$ on $X^{g}$.

Definition 2.3. The $g$-localised $\hat{A}$-genus of $X$ is

$$
\hat{A}_{g}(X):=\int_{X^{g}} c^{g} \frac{\hat{A}\left(X^{g}\right)}{\operatorname{det}\left(1-g e^{-R^{\mathcal{N}} / 2 \pi i}\right)^{1 / 2}} .
$$

If $g=e$, then

$$
\hat{A}_{e}(X)=\int_{X} c^{e} \hat{A}(X)
$$

is the $L^{2}-\hat{A}$ genus of $X$ used in [41]. If $G$ is discrete and acts properly and freely on $X$, then $\hat{A}_{e}(X)=\hat{A}(X / G)$.

Corollary 2.4. Suppose that either

- $G$ is any locally compact group and $g=e$;

- $G$ is discrete and finitely generated and $g$ is any element;

- $G$ is a connected semisimple Lie group and $g$ is a semisimple element.

Let $M$ be a manifold on which $G$ acts properly and that admits a $G$-equivariant Spin-structure. Let $H \subset M$ be a $G$-invariant, cocompact hypersurface such that $M \backslash H=X \cup Y$ for disjoint open subsets $X, Y \subset M$. The localised $\hat{A}$-genus $\hat{A}_{g}(H)$ is independent of the choice of a Riemannian metric. If $M$ admits a complete, $G$-invariant Riemannian metric whose scalar curvature is nonnegative, and positive in a neighbourhood of $H$, then $\hat{A}_{g}(H)=0$.

Theorem 2 in [21] is a generalisation of Atiyah and Hirzebruch's vanishing theorem [3] to actions by noncompact groups. It states that if $G$ is a connected Lie group, and not all stabilisers of the action by $G$ on $H$ are maximal compact, then $\operatorname{index}_{G}\left(D^{H}\right)=0$. So in this setting, the obstructions in Theorem 2.1 and Corollary 2.4 vanish. This makes it a natural question whether Riemannian metrics as in Theorem 2.1 exist if $G$ is a connected Lie group. A partial affirmative answer to that question is given in Subsection 2.2 ,

This also means that the natural place to look for examples and applications where Theorem 2.1 and Corollary 2.4 yield nontrivial obstructions is the setting where $G$ has infinitely many connected components. (The vanishing result generalises directly to the case where $G$ has finitely many 
connected components.) As noted in Remark 2.2, Theorem 2.1 implies Theorem A in [9], in the case where $G$ is the fundamental group of $M / G$ and $M$ is its universal cover. More generally, if $G$ is discrete, then $M / G$ is an orbifold. Then Theorem 2.1 and Corollary 2.4 lead to obstructions to orbifold metrics on $M / G$ with nonnegative scalar curvature, and positive scalar curvature near the sub-orbifold $N / G$. If $G$ acts freely, then $\hat{A}_{g}(H)$ is zero if $g \neq e$ (and $\hat{A}_{e}(H)=\hat{A}(H / G)$ ), but in the orbifold case the localised $\hat{A}$-genera for nontrivial elements $g$ are additional obstructions to positive scalar curvature.

2.2. Existence for connected Lie groups. In this subsection, we suppose that $G$ is a connected Lie group. As pointed out at the end of the previous subsection, for such groups the obstructions to $G$-invariant Riemannian metrics with positive scalar curvature in Theorem 2.1 and Corollary 2.4 vanish under a mild assumption on the action, so it is natural to investigate existence of such metrics.

If $G$ is connected, Abels' global slice theorem [1] implies we have a diffeomorphism $M \cong G \times_{K} N$, for a $K$-invariant submanifold $N \subset M$. Our existence result, Theorem 2.9, supposes that such a slice $N$ has $K$-bounded geometry, a notion introduced in Definition 2.8 .

Suppose a compact, connected Lie group $K$ acts isometrically on a complete Riemannian manifold $\left(N, g_{N}\right)$. Let $b$ denote a bi-invariant Riemannian metric on $K$. For each $y \in N$ we have a linear map

$$
\varphi_{y}: \mathfrak{k} \rightarrow T_{y} N
$$

defined by $\varphi_{y}(X):=\left.\frac{d}{d t}\right|_{t=0} \exp (t X) y$, for $X \in \mathfrak{k}$. Define a pointwise norm function

$$
\|\varphi\|: N \rightarrow \mathbb{R}, \quad y \mapsto\left\|\varphi_{y}\right\|,
$$

where $\|\cdot\|$ denotes the linear operator norm with respect to $b$ and $g_{N}$.

Definition 2.5. We say that the action of $K$ on $N$ has no shrinking orbits if, for any neighbourhood $U$ of the fixed point set $N^{K}$, there exists a constant $C_{U}>0$ such that for all $y \in N \backslash U$ we have

$$
\|\varphi(y)\| \geq C_{U}
$$

where the norm function is taken with respect to the Riemannian metric $g$.

We remark that the condition of the action having no shrinking orbits is independent of $b$.

Example 2.6. Suppose that $N=\mathbb{R}^{2}$, on which $K=\mathrm{SO}(2)$ acts in the natural way. Let $\psi \in C^{\infty}\left(\mathbb{R}^{2}\right)$ be positive and rotation-invariant, and consider the Riemannian metric on $\mathbb{R}^{2}$ equal to $\psi^{2}$ times the Euclidean metric. Then for all $y \in \mathbb{R}^{2}$ and $X \in \mathbb{R} \cong \mathfrak{s o}(2)$,

$$
\varphi_{y}(X)=X\left(\begin{array}{cc}
0 & -1 \\
1 & 0
\end{array}\right) y
$$


So $\|\varphi\|(y)=\psi(y)\|y\|$, where $\|y\|$ is the Euclidean norm of $y$. Hence the action has no shrinking orbits if and only if the function $y \mapsto \psi(y)\|y\|$ has a positive lower bound outside a neighbourhood of $\left(\mathbb{R}^{2}\right)^{\mathrm{SO}(2)}=\{0\}$.

Now let us define the notion of $K$-bounded geometry, which is a strengthening of the standard notion of bounded geometry.

Definition 2.7. A Riemannian manifold has bounded geometry if

- its injectivity radius is positive;

- for each $l \geq 0$ there exists $C_{l}>0$ such that $\left\|\nabla^{l} R\right\|_{\infty} \leq C_{l}$, where $R$ is the Riemann curvature tensor.

Definition 2.8. The action of $K$ on $N$ is said to have $K$-bounded geometry if it has no shrinking orbits and $N$ has bounded geometry.

Our main existence result, proved in Subsection 6.2, is the following.

Theorem 2.9. Let $G$ be a connected Lie group, and $K<G$ a maximal compact subgroup with non-abelian identity component. Let $N$ be a manifold admitting an effective action by $K$ with compact fixed point set. If there exists a Riemannian metric on $N$ such that the $K$-action has $K$-bounded geometry, then the manifold $G \times_{K} N$ admits a $G$-invariant metric with uniformly positive scalar curvature.

This result may be viewed as a strengthening of the vanishing of the obstructions to $G$-invariant metrics of positive scalar curvature in Theorem 2.1 and Corollary 2.4 in the case of connected Lie groups, by the result in [21], in the same way that Lawson and Yau's [27] construction of metrics of positive scalar curvature strengthens the vanishing of the $\hat{A}$-genus as in Atiyah and Hirzebruch's vanishing theorem [3] in the compact case. (See the diagram on page 233 of [27].)

Remark 2.10. By Abels' slice theorem [1, every manifold with a proper action by a connected Lie group $G$ is of the form $G \times_{K} N$ in Theorem 2.9. The condition that $N^{K}$ is compact is equivalent to the condition that the points in $G \times_{K} N$ whose stabilisers in $G$ are maximal compact form a cocompact set.

\section{AN INDEX THEOREM}

We will deduce Theorem 2.1, and hence Corollary 2.4, from an equivariant index theorem for Callias-type operators, Theorem 3.4. This is based on equivariant index theory for such operators with respect to proper actions, developed in [15]. The proof of the index theorem involves several arguments analogous to those in [9].

3.1. The $G$-Callias-type index. From now on, $M$ will be a complete Riemannian manifold on which $G$ acts properly and isometrically. Let $S \rightarrow M$ 
denote a $\mathbb{Z} / 2$-graded, $G$-equivariant Clifford module over $M$, and $D$ an oddgraded Dirac operator on $\Gamma^{\infty}(S)$, associated to a $G$-invariant Clifford connection on $S$ via the Clifford action by $T M$ on $S$.

Let $\Phi$ be an odd, $G$-equivariant, fibrewise Hermitian vector bundle endomorphism of $S$.

Definition 3.1. The endomorphism $\Phi$ is admissible for $D$ if

- the operator $D \Phi+\Phi D$ on $\Gamma^{\infty}(S)$ is a vector bundle endomorphism; and

- there are a cocompact subset $Z \subset M$ and a constant $C>0$ such that we have the pointwise estimate

$$
\Phi^{2} \geq\|D \Phi+\Phi D\|+C
$$

on $M \backslash Z$.

In this setting the operator $D+\Phi$ is called a $G$-Callias-type operator.

In the rest of the paper, we will use the following notation. Let $\mu$ denote the modular function on $G$. Let $C^{*}(G)$ be either the reduced or maximal group $C^{*}$-algebra of $G$. Let $G$ act on sections of the bundle $S$ by

$$
(g s)(x):=g\left(s\left(g^{-1} x\right)\right)
$$

for $s$ a section, $g \in G$ and $x \in M$.

Equip the space $\Gamma_{c}(S)$ with a right $C_{c}(G)$-action defined by

$$
\left(s_{1} \cdot b\right)(x):=\int_{G}(g s)(x) \cdot b\left(g^{-1}\right) \mu(g)^{-1 / 2} d g
$$

and a $C_{c}(G)$-valued inner product defined by

$$
\left(s_{1}, s_{2}\right)_{C^{*}(G)}(g):=\mu(g)^{-1 / 2}\left(s_{1}, g s_{2}\right)_{L^{2}(S)},
$$

for $s_{1}, s_{2} \in \Gamma_{c}^{\infty}(S), b \in C_{c}(G), g \in G$, and $x \in M$. Let $\mathcal{E}$ be the Hilbert $C^{*}(G)$-module completion of $\Gamma_{c}^{\infty}(S)$ with respect to this structure.

The definition of the equivariant index of $G$-Callias-type operators is based on the following result, Theorem 4.19 in [15].

Theorem 3.2. There is a continuous G-invariant cocompactly supported function $f$ on $M$ such that

$$
F:=(D+\Phi)\left((D+\Phi)^{2}+f\right)^{-1 / 2},
$$

is a well-defined, adjointable operator on $\mathcal{E}$, such that $(\mathcal{E}, F)$ is a Kasparov $\left(\mathbb{C}, C^{*}(G)\right)$-cycle. Its class in $K K\left(\mathbb{C}, C^{*}(G)\right)$ is independent of the function $f$ chosen.

For details about the definition of the operator $F$, we refer to Definition 4.11 in $[15$.

Definition 3.3. The $G$-index of the $G$-Callias-type operator $D+\Phi$ is the class

$$
\operatorname{index}_{G}(D+\Phi):=[\mathcal{E}, F] \in K_{0}\left(C^{*}(G)\right)=K K\left(\mathbb{C}, C^{*}(G)\right)
$$

as in Theorem 3.2 , 
3.2. Hypersurfaces and the index theorem. In the setting of the previous subsection, we now suppose that $S=S_{0} \oplus S_{0}$ for an ungraded, $G$ equivariant Clifford module $S_{0}$ over $M$, where the first copy of $S_{0}$ is the even part of $S$, and the second copy is the odd part. Suppose that

$$
D=\left(\begin{array}{cc}
0 & D_{0} \\
D_{0} & 0
\end{array}\right)
$$

for a Dirac operator $D_{0}$ on $S_{0}$, and that

$$
\Phi=\left(\begin{array}{cc}
0 & i \Phi_{0} \\
-i \Phi_{0} & 0
\end{array}\right)
$$

for a Hermitian endomorphism $\Phi_{0}$ of $S_{0}$. (The conditions on $D \Phi+\Phi D$ then become conditions on $\left[D_{0}, \Phi_{0}\right]$.)

Let $Z$ be as in Definition 3.1. Let $M_{-} \subset M$ be a $G$-invariant, cocompact subset containing $Z$ in its interior, such that $N:=\partial M_{-}$is a smooth submanifold of $M$. Let $M_{+}$be the closure of the complement of $M_{-}$, so that $N=M_{-} \cap M_{+}$and $M=M_{-} \cup M_{+}$. In this and similar settings, we write

$$
M=M_{-} \cup_{N} M_{+} .
$$

By (3.1), the restriction of $\Phi_{0}$ to $N$ is fibrewise invertible. Let $S_{+}^{N} \rightarrow$ $N$ and $S_{-}^{N} \rightarrow N$ be its positive and negative eigenbundles. (These are vector bundles, even though eigenbundles for single eigenvalues may not be.) Clifford multiplication by the unit normal vector field $\hat{n}$ to $N$ pointing into $M_{+}$, times $-i$, defines $G$-invariant gradings on $S_{ \pm}^{N}$.

Let $\nabla^{S_{0}}$ be the Clifford connection on $S_{0}$ used to define $D_{0}$. By restriction and projection, it defines connections $\nabla^{S_{ \pm}^{N}}$ on $S_{ \pm}^{N}$. The Clifford action by $\left.T M\right|_{N}$ on $\left.S_{0}\right|_{N}$ preserves $S_{ \pm}^{N}$ by the first condition in Definition 3.1, see also Remark 1.2 in [2]. Hence the connections $\nabla^{S_{ \pm}^{N}}$ define Dirac operators $D^{S_{ \pm}^{N}}$ on $\Gamma^{\infty}\left(S_{ \pm}^{N}\right)$. These operators are odd-graded. Because $N$ is cocompact, $D^{S_{+}^{N}}$ has an equivariant index

$$
\operatorname{index}_{G}\left(D^{S_{+}^{N}}\right) \in K_{0}\left(C^{*}(G)\right)
$$

defined by the analytic assembly map [4].

Theorem 3.4 (G-Callias-type index theorem). We have

$$
\operatorname{index}_{G}(D+\Phi)=\operatorname{index}_{G}\left(D^{S_{+}^{N}}\right) \quad \in K_{0}\left(C^{*}(G)\right) .
$$

Versions of this result where $G$ is trivial were proved in [2, 5, 7, 8, 25]. Versions for operators on bundles of modules over operator algebras are proved in 6, 9].

There are various index theorems for the the image of the right hand side of (3.7) under traces [24, 41, 40] or pairings with higher cyclic cocycles [23, 33, 34]. Via these results, Theorem 3.4 yields topological expressions for the corresponding images of the left hand side of (3.7). The results in [24, 41, 40] will be used to deduce Corollary 2.4 from Theorem 2.1. 


\section{Properties of the $G$-Callias-type index}

To prove Theorem 3.4, we will make use of the properties of the index of Definition 3.3 that we describe below.

4.1. Sobolev modules. We start by recalling the definition of Sobolev Hilbert $C^{*}(G)$-modules from [15]. Let $M, G, S$ and $D$ be as in Subsection 3.1 .

Definition 4.1. For each nonnegative integer $j$, define $\Gamma_{c}^{\infty, j}(S)$ to be the pre-Hilbert $C_{c}(G)$-module whose underlying vector space is $\Gamma_{c}^{\infty}(S)$, equipped with the right $C_{c}(G)$-action defined by $(3.2)$, and $C_{c}(G)$-valued inner product defined by

$$
\left\langle e_{1}, e_{2}\right\rangle_{\mathcal{E}^{j}}=\sum_{k=0}^{j}\left(D^{k} e_{1}, D^{k} e_{2}\right)_{C^{*}(G)},
$$

where $e_{1}, e_{2} \in \Gamma_{c}^{\infty}(S)$ and $(-,-)_{C^{*}(G)}$ is as in 3.3 . Here we set $D^{0}$ equal to the identity operator. Denote by $\mathcal{E}^{j}(S)=\mathcal{E}^{j}$ the vector space completion of $\Gamma_{c}^{\infty, j}(S)$ with respect to the norm induced by $\langle-,-\rangle_{\mathcal{E}^{j}}$, and extend naturally the $C_{c}^{\infty}(G)$-action to a $C^{*}(G)$-action, and $\langle-,-\rangle_{\mathcal{E}^{j}}$ to a $C^{*}(G)$-valued inner product on $\mathcal{E}^{j}$, to give it the structure of a Hilbert $C^{*}(G)$-module. We call $\mathcal{E}^{j}$ the $j$-th $G$-Sobolev module with respect to $D$.

The module $\mathcal{E}$ defined above Theorem 3.2 equals $\mathcal{E}^{0}$. The following version of the Rellich lemma holds for Sobolev modules (Theorem 3.12 in [15]).

Theorem 4.2. Let $f$ be a continuous $G$-invariant cocompactly supported function on $M$. Then multiplication by $f$ defines an element of $\mathcal{K}\left(\mathcal{E}^{s}, \mathcal{E}^{t}\right)$ whenever $s>t$.

We will state and prove a homotopy invariance result, Proposition 4.9, for the index in Definition 3.3, that will be of use later. A hypothesis in this result is that a certain vector bundle endomorphism defines adjointable operators on the Sobolev modules $\mathcal{E}^{0}$ and $\mathcal{E}^{1}$. In order to check this condition in some geometric situations relevant to us, we will need Propositions 4.3 and 4.4 below.

Proposition 4.3. A smooth, $G$-invariant, uniformly bounded bundle endomorphism of $S$ defines an element of $\mathcal{L}\left(\mathcal{E}^{0}\right)$.

Proof. Let $\Psi$ be a smooth, $G$-invariant, uniformly bounded bundle endomorphism of $S$. Since $\Psi$ is uniformly bounded, it defines a bounded operator on $L^{2}(S)$. Let $\|\Psi\|$ denote its operator norm, let $c$ be a cutoff function on $M$, and let $\Psi^{*}$ be the pointwise adjoint of $\Psi$. Since the operator $\Psi_{1}:=\Psi^{*} \Psi-\|\Psi\|^{2}$ is positive on $L^{2}(S)$, it has a positive square root $Q$ that one observes is $G$-invariant. For a fixed $e \in \Gamma_{c}^{\infty}(S)$, the function

$$
g \mapsto\left(c \Psi_{1}(g e), g e\right)_{L^{2}(S)}=(\sqrt{c} Q(g e), \sqrt{c} Q(g e))_{L^{2}(S)}
$$

has compact support in $G$, by properness of the $G$-action. Thus the map $G \rightarrow L^{2}(S)$ defined by $g \mapsto \sqrt{c} Q(g e)$ has compact support in $G$. 
It follows that for any unitary representation $\pi$ of $G$ on a Hilbert space $H$ and $h \in H$,

$$
v:=\int_{G} \mu(g)^{-1 / 2} \sqrt{c} Q(g e) \otimes \pi(g) h d g
$$

is a well-defined vector in $L^{2}(S) \otimes H$. By computations similar to those in the proof of Proposition 5.4 in [15], one sees that $\|v\|_{L^{2}(S) \otimes H}$ equals

$$
\int_{G} \int_{G}\left\langle g c \Psi_{1} g^{-1} e, e\right\rangle_{\mathcal{E}^{0}}\left(g^{\prime}\right) d g \cdot\left(\pi\left(g^{\prime}\right) h, h\right)_{H} d g^{\prime} .
$$

Thus, for any unitary representation $\pi$ of $G$,

$$
\pi\left(\int_{G}\left\langle g c \Psi_{1} g^{-1} e, e\right\rangle_{\mathcal{E}^{0}} d g\right)=\pi\left(\left\langle\Psi_{1} e, e\right\rangle_{\mathcal{E}^{0}}\right)
$$

is a positive operator, where we let $f \in C_{c}(G)$ act on $H$ by

$$
\pi(f) h:=\int_{G} f(g) \pi(g) h d g .
$$

It follows that the element

$$
\left\langle\Psi_{1} e, e\right\rangle_{\mathcal{E}^{0}}=\left\langle\left(\Psi^{*} \Psi-\|\Psi\|^{2}\right) e, e\right\rangle_{\mathcal{E}^{0}}=\langle\Psi e, \Psi e\rangle_{\mathcal{E}^{0}}-\|\Psi\|^{2}\langle e, e\rangle_{\mathcal{E}^{0}} .
$$

is positive in $C^{*}(G)$. Hence $\Psi$ extends to an operator on all of $\mathcal{E}^{0}$. Similarly, $\Psi^{*}$ defines an operator on all of $\mathcal{E}^{0}$ that one checks is the adjoint of $\Psi$.

Proposition 4.4. Suppose that there are a G-invariant, cocompact subset $K \subset M$ and $a G$-invariant, cocompact hypersurface $N \subset M$ such that there is a $G$-equivariant isometry $M \backslash K \cong N \times(0, \infty)$, and a $G$-equivariant vector bundle isomorphism $\left.\left.S\right|_{M \backslash K} \cong S\right|_{N} \times(0, \infty)$. Let $\Psi$ be a $G$-equivariant vector bundle endomorphism of $S$. Suppose that, on $M \backslash K, \Psi$ and $D$ are constant in the factor $(0, \infty)$ of $M \backslash K \cong N \times(0, \infty)$. Then $\Psi$ defines an element of $\mathcal{L}\left(\mathcal{E}^{1}\right)$.

The proof uses the next lemma. To state it, let $H^{1}(S)$ be the completion of $\Gamma_{c}^{\infty}(S)$ with respect to the inner product

$$
(-,-)_{H^{1}(S)}=(-,-)_{L^{2}(S)}+(D-, D-)_{L^{2}(S)} .
$$

Lemma 4.5. Let $M$ and $S$ be as in Proposition 4.4. Let $\Theta$ be a bounded, positive operator on $H^{1}(S)$ such that

- $\Theta$ preserves the subspace $\Gamma_{c}^{\infty}(S)$;

- for any e $\in \Gamma_{c}^{\infty}(S)$, the function $a: G \rightarrow \mathbb{R}$ given by $g \mapsto(\Theta(g e), g e)_{H^{1}(S)}$ has compact support in $G$.

Then

$$
\int_{G}\left\langle g \Theta g^{-1} e, e\right\rangle_{\mathcal{E}^{1}} d g
$$

is a positive element of $C^{*}(G)$. 
Proof. Let $Q$ be the positive square root of $\Theta$ in $\mathcal{B}\left(H^{1}(S)\right)$. Since $a$ has compact support, and $(\Theta(g e), g e)_{H^{1}(S)}=(Q(g e), Q(g e))_{H^{1}(S)}$, the map $G \rightarrow$ $H^{1}(S)$ defined by $g \mapsto Q(g e)$ has compact support in $G$. As in the proof of Proposition 4.3, one finds that for any unitary representation $\pi$ of $G$ on a Hilbert space $H$ and $h \in H$,

$$
v:=\int_{G} \mu(g)^{-1 / 2} Q(g e) \otimes \pi(g) h d g
$$

is a well-defined vector in $H^{1}(S) \otimes H$, and that

$$
\int_{G} \int_{G}\left\langle g Q^{2} g^{-1} e, e\right\rangle_{\mathcal{E}^{1}}\left(g^{\prime}\right) d g \cdot\left(\pi\left(g^{\prime}\right) h, h\right)_{H} d g^{\prime}=\|v\|_{H^{1}(S) \otimes H} \geq 0 .
$$

Similarly to the proof of Proposition 4.3 , we deduce that $\int_{G}\left\langle g \Theta g^{-1} e, e\right\rangle_{\mathcal{E}^{1}} d g$ is a positive element of $C^{*}(G)$.

Proof of Proposition 4.4. Because of the forms of $M$ and $S$, there is a canonical (up to equivalence) first Sobolev norm $\|\cdot\|_{1}$ on sections of $S$ that is $G$-invariant, and invariant under the relevant class of translations in the factor $(0, \infty)$ of $N \times(0, \infty)$. Because $\Psi$ is an order zero differential operator constant on the factor $(0, \infty)$, it defines a bounded operator with respect to $\|\cdot\|_{1}$. Due to the form of $D$, the norm on $H^{1}(S)$ is equivalent to $\|\cdot\|_{1}$, and so $\Psi$ defines a bounded operator on $H^{1}(S)$.

Let $\pi_{N}: M \backslash K \cong N \times(0, \infty) \rightarrow N$ be the natural projection. Let $c$ be a cutoff function on $M$ such that

$$
\left.c\right|_{M \backslash K}=\pi_{N}^{*} c_{N}
$$

for a cutoff function $c_{N}$ on $N$. Let $\Psi^{*}$ and $c^{*}$ denote the respective adjoints of $\Psi$ and $c$ in $\mathcal{B}\left(H^{1}(S)\right)$. Then the operator

$$
\Psi_{1}:=\frac{c \Psi^{*} \Psi+\Psi^{*} \Psi c^{*}}{2}
$$

is bounded and self-adjoint on $H^{1}(S)$ with norm at most $\|\Psi\|^{2}\|c\|$, where the norms are taken in $\mathcal{B}\left(H^{1}(S)\right)$.

Let $c^{\prime}$ be a smooth, nonnegative function on $M$ that is identically 1 on the support of $c$, and such that

$$
\left.c^{\prime}\right|_{M \backslash K}=\pi_{N}^{*} c_{N}^{\prime}
$$

for a compactly supported function $c_{N}^{\prime}$ on $N$. Consider the endomorphism $\Psi_{2}:=\left(c^{\prime}\right)^{*} c^{\prime}\|\Psi\|^{2}\|c\|-\Psi_{1}$ of $S$. For the same reasons as for $\Psi$, it defines a bounded operator on $H^{1}(S)$. Fix $e \in \Gamma_{c}^{\infty}(S)$. Because $\Psi$ is a positive bounded operator on $H^{1}(S)$, we may apply Lemma 4.5 with $\Theta=\Psi_{2}$ to conclude that

$$
\int_{G}\left\langle\left(g \Psi_{2} g^{-1}\right) e, e\right\rangle_{\mathcal{E}^{1}} d g=\int_{G}\left\langle\left(g\left(\left(c^{\prime}\right)^{*} c^{\prime}\right) g^{-1}\|\Psi\|^{2}\|c\|\right) e, e\right\rangle_{\mathcal{E}^{1}} d g-\left\langle\Psi^{*} \Psi e, e\right\rangle_{\mathcal{E}^{1}}
$$


is positive in $C^{*}(G)$. Define $b: M \times G \rightarrow \mathbb{R}$ by

$$
b(x, g):=b_{x}(g):=c^{\prime}\left(g^{-1} x\right) .
$$

By construction of $c^{\prime}$, the quantities

$$
C_{1}(x):=\int_{G} c^{\prime}\left(g^{-1} x\right)^{2} d g, \quad C_{2}(x):=\left\|d c^{\prime}\right\|_{\infty}^{2} \cdot \operatorname{vol}\left(\operatorname{supp}_{G}\left(b_{x}\right)\right)
$$

are bounded as functions of $x \in M$ (see also Remark 4.6 below). A direct calculation shows that

$$
\begin{aligned}
\left\|\int_{G}\left\langle\left(g\left(\left(c^{\prime}\right)^{*} c^{\prime}\right) g^{-1}\|\Psi\|^{2}\|c\|\right) e, e\right\rangle_{\mathcal{E}^{1}} d g\right\|_{C^{*}(G)} & =\|\Psi\|^{2}\|c\|\left\|\int_{G}\left\langle c^{\prime} g^{-1} e, c^{\prime} g^{-1} e\right\rangle_{\mathcal{E}^{1}} d g\right\|_{C^{*}(G)} \\
& \leq\|\Psi\|^{2}\|c\|\left(\left\|C_{1}\right\|_{\infty}\|e\|_{\mathcal{E}^{1}}^{2}+\left\|C_{2}\right\|_{\infty}\|e\|_{\mathcal{E}^{0}}^{2}\right) \\
& \leq C_{3}\|\Psi\|^{2}\|e\|_{\mathcal{E}^{1}}^{2},
\end{aligned}
$$

for some constant $C_{3}$. Together with positivity of 4.1), this implies that

$$
\|\Psi e\|_{\mathcal{E}^{1}}^{2}=\left\|\left\langle e, \Psi^{*} \Psi e\right\rangle_{\mathcal{E}^{1}}\right\|_{C^{*}(G)} \leq C_{3}\|\Psi\|^{2}\|e\|_{\mathcal{E}^{1}}^{2},
$$

so that $\Psi$ extends to an operator on all of $\mathcal{E}^{1}$. Similarly, the $H^{1}(S)$-adjoint $\Psi^{*}$ defines an operator on $\mathcal{E}^{1}$ that one checks is the adjoint of $\Psi$.

Remark 4.6. As can be seen from the proof, the conclusion of Proposition 4.4 holds more broadly for any $M$ on which the functions $C_{1}$ and $C_{2}$ on $M$ are bounded.

4.2. Vanishing. Two cases where the index of Definition 3.3 vanishes are straightforward to prove, but we state them here because they will be used in various places.

Lemma 4.7. If (3.1) holds on all of $M$, then $\operatorname{index}_{G}(D+\Phi)=0$.

Proof. In this setting, the operator $F$ in (3.4) is invertible. This implies that the $K K$-cycle $(\mathcal{E}, F)$ is operator homotopic to the degenerate cycle $\left(\mathcal{E}, F\left(F^{*} F\right)^{-1 / 2}\right)$.

Lemma 4.8. If $M / G$ is compact, and $D$ and $\Phi$ are of the forms (3.5) and (3.6), then $\operatorname{index}_{G}(D)=0$.

Proof. In this setting, $\Phi$ is bounded, and the cycle $(\mathcal{E}, F)$ is operator homotopic to $\left(\mathcal{E}, \frac{D}{\sqrt{D^{2}+1}}\right)$.

In general, let $A$ be a $C^{*}$-algebra, let $\mathcal{E}_{0}$ be a Hilbert $A$-module, and set $\mathcal{E}:=\mathcal{E}_{0} \oplus \mathcal{E}_{0}$. and an adjointable operator $F$ on $\mathcal{E}$ such that $(\mathcal{E}, F)$ is a Kasparov $(\mathbb{C}, A)$-cycle, and $F$ is of the form

$$
F=\left(\begin{array}{cc}
0 & F_{0} \\
F_{0} & 0
\end{array}\right)
$$

for a (necessarily self-adjoint) $F_{0} \in \mathcal{L}\left(\mathcal{E}_{0}\right)$. Then $[\mathcal{E}, F]=0 \in K_{0}(A)$. Because $\frac{D}{\sqrt{D^{2}+1}}$ is of the form 4.2 , the claim follows. 
4.3. Homotopy invariance. The index of Definition 3.3 has a homotopy invariance property analogous to Proposition 4.1 in [9]. This homotopy invariance applies in a more general setting than Callias-type operators.

Let $P$ be an odd, $G$-equivariant Dirac-type operator on a $\mathbb{Z} / 2$-graded Clifford module $\mathcal{S}$, and let $\Psi$ be an odd, smooth $G$-equivariant, uniformly bounded Hermitian vector bundle endomorphism of $\mathcal{S}$. Fix $t_{0}<t_{1} \in \mathbb{R}$. For $t \in\left[t_{0}, t_{1}\right]$, consider the operator $P_{t}:=P+t \Psi$.

Proposition 4.9 (Homotopy invariance). Suppose that

(1) for $j=0,1$, the endomorphism $\Psi$ defines an adjointable operator on the Sobolev module $\mathcal{E}^{j}$ of Definition 4.1;

(2) there is a nonnegative, $G$-invariant, cocompactly supported function $f \in C^{\infty}(M)$ such that for all $t \in\left[t_{0}, t_{1}\right]$, the operator $P_{t}^{2}+f: \mathcal{E}^{2} \rightarrow$ $\mathcal{E}^{0}$ is invertible, with inverse in $\mathcal{L}\left(\mathcal{E}^{0}, \mathcal{E}^{2}\right)$.

Then $\operatorname{index}_{G}\left(P_{t}\right) \in K_{0}\left(C^{*}(G)\right)$ is independent of $t \in\left[t_{0}, t_{1}\right]$.

Proof. The proof proceeds identically to the proof of Proposition 4.1 in [9], with the exceptions that Theorem 4.2 should be substituted for Lemma 4.2 in [9], and Lemmas 4.4 and 4.6(a) in [15] should be substituted for Lemmas 1.4 and 1.5 in [7], respectively.

Corollary 4.10. If $D+\Phi$ is a $G$-Callias-type operator on $S \rightarrow M$, and $\Psi$ is a $G$-equivariant, odd vector bundle endomorphism of $S$ that equals zero outside a cocompact set, then $\operatorname{index}_{G}(D+\Phi)=\operatorname{index}_{G}(D+\Phi+\Psi)$.

Proof. We set $P=D+\Phi$ and apply Proposition 4.9. Since $\Psi$ is cocompactly supported, the first condition in Proposition 4.9 holds by Proposition 3.5 in [15]. For the same reason, $\Phi+t \Psi$ is a Callias-type potential for all $t \in \mathbb{R}$, so by Theorem 5.6 in [15, the second condition in Proposition 4.9 holds for $t \in[0,1]$, where a priori the function $f$ may depend on $t$. But since $\Psi$ is zero outside a cocompact set, we can choose $f$ independent of $t$. The claim then follows from Proposition 4.9

Remark 4.11. In Proposition 4.9, it is not assumed that $\Psi$ is a Callias-type potential in the sense of Definition 3.1. We will use Proposition 4.9 in this greater generality in the proof of Lemma 5.2 .

Remark 4.12. Corollary 4.10 can be used to give an alternative proof of Lemma 4.7 this corollary implies that in the setting of that lemma,

$\operatorname{index}_{G}(D+\Phi)=\operatorname{index}_{G}(D-\Phi)=\operatorname{index}_{G}\left((D+\Phi)^{*}\right)=-\operatorname{index}_{G}(D+\Phi)$.

See also Corollary 4.9 in [9].

4.4. A relative index theorem. We will use an analogue of Bunke's relative index theorem, Theorem 1.2 in [7]. For $j=1,2$, let $M_{j}, S_{j}, D_{j}$ and $\Phi_{j}$, respectively, be as $M, S, D$ and $\Phi$ were before. Suppose that there are a $G$-invariant hypersurface $N_{j} \subset M_{j}$ and a $G$-invariant tubular neighbourhood $U_{j}$ of $N_{j}$, and that there is a $G$-equivariant isometry $\psi: U_{1} \rightarrow U_{2}$ such that 
- $\psi\left(N_{1}\right)=N_{2}$;

- $\left.\psi^{*}\left(\left.S_{2}\right|_{U_{2}}\right) \cong S_{1}\right|_{U_{1}}$

- $\psi^{*}\left(\left.\nabla^{S_{2}}\right|_{U_{2}}\right)=\left.\nabla^{S_{1}}\right|_{U_{1}}$, where $\nabla^{S_{j}}$ is the Clifford connection used to define $D_{j}$; and

- $\left.\Phi_{1}\right|_{U_{1}}$ corresponds to $\left.\Phi_{2}\right|_{U_{2}}$ via $\psi$.

Suppose that $M_{j}=X_{j} \cup_{N_{j}} Y_{j}$ for closed, $G$-invariant subsets $X_{j}, Y_{j} \subset M_{j}$. We identify $N_{1}$ and $N_{2}$ via $\psi$ and write $N$ for this manifold when we do not want to distinguish between $N_{1}$ and $N_{2}$. Using the map $\varphi$, form

$$
M_{3}:=X_{1} \cup_{N} Y_{2} ; \quad M_{4}:=X_{2} \cup_{N} Y_{1} .
$$

For $j=3,4$, let $S_{j}, D_{j}$ and $\Phi_{j}$ be obtained from the corresponding data on $M_{1}$ and $M_{2}$ by cutting and gluing along $U_{1} \cong U_{2}$ via $\psi$.

Theorem 4.13. In the above situation, $\operatorname{index}_{G}\left(D_{1}+\Phi_{1}\right)+\operatorname{index}_{G}\left(D_{2}+\Phi_{2}\right)=\operatorname{index}_{G}\left(D_{3}+\Phi_{3}\right)+\operatorname{index}_{G}\left(D_{4}+\Phi_{4}\right)$.

Proof. This proof is an adaptation of the proof of Theorem 1.14 in [7], with some results from [15] added. For $j=1,2,3,4$, let $\mathcal{E}_{j}$ and $F_{j}$ be as $\mathcal{E}$ and $F$ above and in Theorem 3.2 , for the data indexed by $j$. Using superscripts op to denote opposite gradings, we write $\mathcal{E}:=\mathcal{E}_{1} \oplus \mathcal{E}_{2} \oplus \mathcal{E}_{3}^{\text {op }} \oplus \mathcal{E}_{4}^{\text {op }}$ and $F:=F_{1} \oplus F_{2} \oplus F_{3} \oplus F_{4}$. We will show that

$$
[\mathcal{E}, F]=0 \quad \in K K_{0}\left(\mathbb{C}, C^{*}(G)\right),
$$

which is equivalent to the theorem.

For $j=1,2$, let $\chi_{X_{j}}, \chi_{Y_{j}} \in C^{\infty}\left(M_{j}\right)$ be real-valued functions such that

- $\operatorname{supp}\left(\chi_{X_{j}}\right) \subset X_{j} \cup U_{j}$ and $\operatorname{supp}\left(\chi_{Y_{j}}\right) \subset Y_{j} \cup U_{j}$;

- $\psi^{*}\left(\left.\chi_{X_{2}}\right|_{U_{2}}\right)=\left.\chi_{X_{1}}\right|_{U_{1}}$ and $\psi^{*}\left(\left.\chi_{Y_{2}}\right|_{U_{2}}\right)=\left.\chi_{Y_{1}}\right|_{U_{1}} ;$ and

- $\chi_{X_{j}}^{2}+\chi_{Y_{j}}^{2}=1$.

We view pointwise multiplication by these functions as operators

$$
\begin{array}{ll}
\chi_{X_{1}}: \mathcal{E}_{1} \rightarrow \mathcal{E}_{3} ; & \chi_{Y_{1}}: \mathcal{E}_{2} \rightarrow \mathcal{E}_{3} ; \\
\chi_{X_{2}}: \mathcal{E}_{1} \rightarrow \mathcal{E}_{4} ; & \chi_{Y_{2}}: \mathcal{E}_{2} \rightarrow \mathcal{E}_{4} .
\end{array}
$$

The adjoints of these operators map in the opposite directions, and are also given by pointwise multiplication by the respective functions. Using these multiplication operators, and the grading operator $\gamma$, we form the operator

$$
X:=\gamma\left(\begin{array}{cccc}
0 & 0 & -\chi_{X_{1}}^{*} & \chi_{X_{2}}^{*} \\
0 & 0 & -\chi_{Y_{1}}^{*} & \chi_{Y_{2}}^{*} \\
\chi_{X_{1}} & \chi_{Y_{1}} & 0 & 0 \\
\chi_{X_{2}} & -\chi_{Y_{2}} & 0 & 0
\end{array}\right)
$$

on $\mathcal{E}$. Then $X$ is an odd, self-adjoint, adjointable operator on $\mathcal{E}$ such that $X^{2}=1$. As such, it generates a Clifford algebra $\mathrm{Cl}$.

We claim that $X F+F X$ is a compact operator. This is based on the Rellich lemma for Hilbert $C^{*}(G)$-modules, Theorem 4.2 , Let $\chi$ be one of the functions $\chi_{X_{j}}$ or $\chi_{Y_{j}}$, viewed as an operator from $\mathcal{E}_{k}$ to $\mathcal{E}_{l}$ as in 4.4. Let $f_{j} \in C^{\infty}(M)$ be as in Theorem 3.2 , for the operator $D_{j}+\Phi_{j}$. For 
$j=1,2,3,4$ and $\lambda \in \mathbb{R}$, the operator $\left(D_{j}^{2}+f_{j}^{2}+\lambda^{2}\right)$ on $\mathcal{E}_{j}$ is invertible by Lemma 4.6 in [15], and we denote its inverse by $R_{j}(\lambda)$.

Then, as in the proof of Theorem 1.14 in [7], and using Proposition 4.12 in [15, we find that the operator

$$
\chi^{*} \circ F_{l}-F_{k} \circ \chi^{*}: \mathcal{E}_{l} \rightarrow \mathcal{E}_{k}
$$

equals

$$
\begin{aligned}
& \quad \frac{2}{\pi} \int_{\mathbb{R}}\left(-\operatorname{grad}(\chi) R_{l}(\lambda)+D_{k}^{2} R_{k}(\lambda) \operatorname{grad}(\chi) R_{l}(\lambda)+\right. \\
& \left.D_{k} R_{k}(\lambda) \operatorname{grad}\left(f_{k}\right) R_{k}(\lambda) \operatorname{grad}(\chi) R_{l}(\lambda)+D_{k} R_{k}(\lambda) \operatorname{grad}(\chi) D_{l} R_{l}(\lambda)\right) d \lambda .
\end{aligned}
$$

Theorem 4.2, together with Lemma 4.6(a) in [15], implies that for all cocompactly supported continuous functions $\varphi$ on $M_{j}$, the compositions $\varphi D_{j}^{n} R_{j}(\lambda)$, $D_{j}^{n} \varphi R_{j}(\lambda)$ and $D_{j}^{n} R_{j}(\lambda) \varphi$ are compact operators on $\mathcal{E}_{j}$ if $n=0,1$, and adjointable operators if $n=1$. So all the terms in the integrand in (4.6) are compact operators. By Lemmas 4.6 and 4.8 in [15], the norm of the integrand in (4.6) is bounded by $a\left(b+\lambda^{2}\right)^{-1}$ for constants $a, b>0$. So the integral converges in the operator norm on $\mathcal{L}(\mathcal{E})$, and we conclude that 4.5 is a compact operator on $\mathcal{E}$. This implies that $X F+F X$ is a compact operator.

Because $X$ generates $\mathrm{Cl}$ and $X$ anticommutes with $F$ modulo compacts, the pair $(\mathcal{E}, F)$ is a Kasparov $\left(\mathrm{Cl}, C^{*}(G)\right)$-cycle. Its class in $K K\left(\mathrm{Cl}, C^{*}(G)\right)$ is mapped to the left-hand aside of 4.3 by the pullback along the inclusion map $\mathbb{C} \hookrightarrow \mathrm{Cl}$. That map is zero by Lemma 1.15 in [7, so 4.3 follows.

Theorem 4.13 implies the following version of Proposition 5.9 in [9].

Corollary 4.14. In the setting of Theorem 4.13, suppose that for $j=1,2$, the set $X_{j}$ is cocompact, and contains a set $Z_{j}$ for $\Phi_{j}$ as in Definition 3.1. Then

$$
\operatorname{index}_{G}\left(D_{1}+\Phi_{1}\right)=\operatorname{index}_{G}\left(D_{2}+\Phi_{2}\right) .
$$

Proof. This fact can be deduced from Theorem 4.13 in exactly the same way Proposition 5.9 in [9] is deduced from Theorem 5.7 in [9. Compared to that proof in [9], references to Corollaries 3.4 and 4.9 and Theorem 5.7 in that paper should be replaced by references to Lemmas 4.7 and 4.8 and Theorem 4.13 , respectively, in the present paper.

The crucial assumption in Corollary 4.14 is that all data near $N_{1}$ can be identified with the corresponding data near $N_{2}$.

\section{Proof of the $G$-Callias-type index theorem}

The first and most important step in the proof of Theorem 3.4 is Proposition 5.1, which states that $\operatorname{index}_{G}(D+\Phi)$ equals the index of a $G$-Calliastype operator on the manifold $N \times \mathbb{R}$, which we will call the cylinder on $N$. 


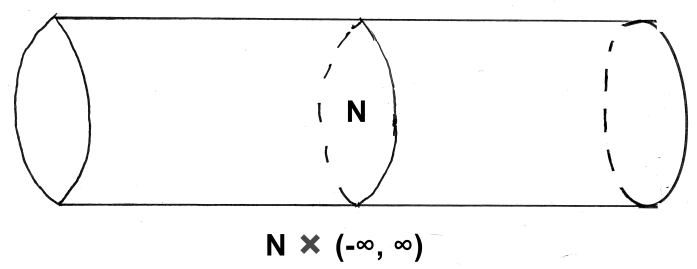

Figure 1 . The cylinder $N \times \mathbb{R}$

See Figure 1. Such an approach is taken in proofs of various other index theorems for Callias-type operators; see for example [2, 6, 7, 9].

In this section, we consider the setting of Subsection 3.2. In particular, $D$ and $\Phi$ are assumed to be of the forms (3.5) and (3.6).

5.1. An index on the cylinder. Let $S_{ \pm}^{N \times \mathbb{R}} \rightarrow N \times \mathbb{R}$ be the pullbacks of the bundles of $S_{ \pm}^{N} \rightarrow N$ defined in Subsection 3.2 along the projection $N \times \mathbb{R} \rightarrow N$. They are Clifford modules, with Clifford actions

$$
\hat{c}(v, t)=c(v+t \hat{n}),
$$

for $v \in T N$ and $t \in \mathbb{R}$, where $c$ is the Clifford action by $T M$ on $S$ (which preserves $S_{+}^{N}$ as pointed out in Subsection 3.2, and $\hat{n}$ is the normal vector field to $N$ in the direction of $M_{+}$. Let $D_{0}^{S_{+}^{N \times R}}$ be the Dirac operator on $\Gamma^{\infty}\left(S_{+}^{N \times \mathbb{R}}\right)$ defined by this Clifford action, and the pullback to $N \times \mathbb{R}$ of the restriction to $N$ of the Clifford connection $\nabla^{S_{+}^{N}}$ used to define $D_{0}^{S_{+}^{N}}$.

Let $\chi \in C^{\infty}(\mathbb{R})$ be an odd function such that $\chi(t)=t$ for all $t \geq 2$. We also denote its pullback to $N \times \mathbb{R}$ by $\chi$. Then pointwise multiplication by

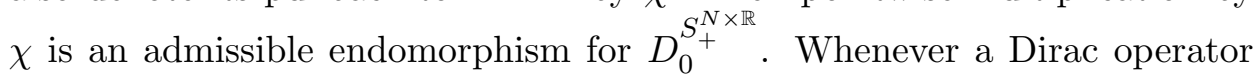
with a subscript 0 is given, we will remove that subscript to denote the corresponding Dirac operator on two copies of the Clifford module in question, as in (3.5). In the current setting, this gives us the Dirac operator

$$
D^{S_{+}^{N \times \mathbb{R}}}=\left(\begin{array}{cc}
0 & D_{0}^{S_{+}^{N \times \mathbb{R}}} \\
D_{0}^{S_{+}^{N \times \mathbb{R}}} & 0
\end{array}\right)
$$

on $\Gamma^{\infty}\left(S_{+}^{N \times \mathbb{R}} \oplus S_{+}^{N \times \mathbb{R}}\right)$. We also consider the admissible endomorphism

$$
\chi^{N \times \mathbb{R}}=\left(\begin{array}{cc}
0 & i \chi \\
-i \chi & 0
\end{array}\right)
$$

of $S_{+}^{N \times \mathbb{R}} \oplus S_{+}^{N \times \mathbb{R}}$ 


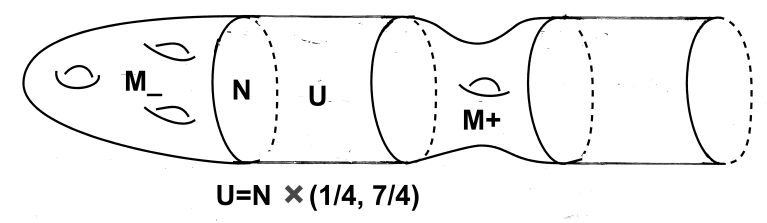

Figure 2. The manifold $M$

Proposition 5.1. We have

$$
\operatorname{index}_{G}(D+\Phi)=\operatorname{index}_{G}\left(D^{S_{+}^{N \times \mathbb{R}}}+\chi^{N \times \mathbb{R}}\right)
$$

The proof of Proposition 5.1 that we give below is an analogue of the proof of Theorem 5.4 in [9]. We give this proof in Subsections 5.2 and 5.3 . referring to [9] for details in some places, and using results from [15] and from Section 4 ,

5.2. Attaching a half-cylinder. Let $S_{0}^{N \times \mathbb{R}} \rightarrow N \times \mathbb{R}$ be the pullback of $\left.S_{0}\right|_{N} \rightarrow N$. We choose $U$ small enough so that $\left.\left.S_{0}\right|_{U} \cong S_{0}^{N \times \mathbb{R}}\right|_{U}$.

Because the sets $X_{j}$ are cocompact in Corollary 4.14 , we initially compare the left-hand side of (5.1) to an index on a manifold where only $M_{+}$is replaced by a half-cylinder $N \times[1, \infty)$. To be more $\operatorname{precise} \operatorname{index}_{G}(D+\Phi)$ is invariant under changes in the Riemannian metric on cocompact sets because the Kasparov ( $\left.\mathbb{C}, C^{*}(G)\right)$-cycles corresponding to two $G$-invariant Riemannian metrics differing only a cocompact set are homotopic by convexity of the space of $G$-invariant Riemannian metrics. We choose a metric such that there is a neighbourhood $U$ of $N$ that is isometric to $N \times(1 / 4,7 / 4)$ (see Figure 2).

By Corollary 4.10, the index of $D+\Phi$ does not change if we change $\Phi_{0}$ in a cocompact set. So we may assume that $\Phi_{0}$ is constant in the direction normal to $N$ inside $U$; i.e. for all $n \in N$ and $t \in(1 / 4,7 / 4), \Phi_{0}(n, t)=\Phi_{0}^{N}(n)$, for an endomorphism $\Phi_{0}^{N}$ of $\left.S_{0}\right|_{N}$. We further choose $U$ such that a set $Z$ as in Definition 3.1 is contained in $M_{-} \backslash U$.

Let $\nabla^{S_{0}^{N \times \mathbb{R}}}$ be the pullback of $\left.\nabla^{S_{0}}\right|_{N}$ to a connection on $S_{0}^{N \times \mathbb{R}}$. We choose the Clifford connection $\nabla^{S_{0}}$ to define $D_{0}$ so that on $U$, it equals the restriction of $\nabla^{S_{0}^{N \times \mathbb{R}}}$ to $N \times(1 / 4,7 / 4)$.

For this structure near $N$, we can form the Riemannian manifold $M_{C}:=$ $M_{-} \cup_{N}[1, \infty)$ (see Figure 3 ), and define the Clifford module $S_{0}^{C} \rightarrow M_{C}$ such that it equals $S_{0}$ on $M_{-}$and $S_{0}^{N \times \mathbb{R}}$ on $N \times(1 / 4, \infty)$. Let $\nabla^{S_{0}^{C}}$ be the Clifford connection on $S_{0}^{C}$ corresponding to $\nabla^{S_{0}}$ on $M_{-}$and to $\nabla^{S_{0}^{N \times \mathbb{R}}}$ on $N \times(1 / 4, \infty)$. Let $D_{0}^{C}$ be the resulting Dirac operator. 


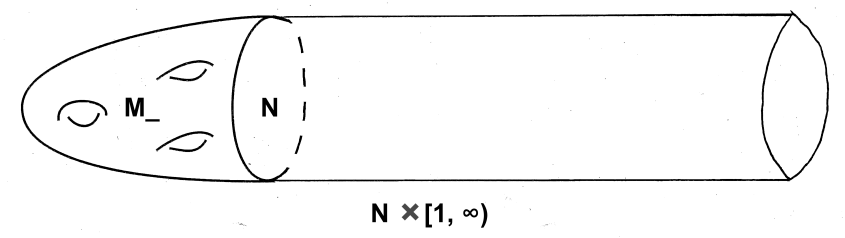

Figure 3 . The manifold $M_{C}$

We define an endomorphism $\Phi_{0}^{C}$ of $S_{0}^{C}$ that is equal to $\Phi_{0}$ on $M_{-}$and to the pullback of $\Phi_{0}^{N}$ on $N \times(1 / 4, \infty)$. Recall that by removing the subscript 0 from $D_{0}^{C}$ we refer to the construction (3.5). Similarly, when we remove the subscript 0 from $\Phi_{0}^{C}$, we will be referring to the endomorphism $\Phi^{C}$ defined by $\Phi_{0}^{C}$ as in (3.6). Then Corollary 4.14 immediately implies that

$$
\operatorname{index}_{G}(D+\Phi)=\operatorname{index}_{G}\left(D^{C}+\Phi^{C}\right) \text {. }
$$

The connection $\nabla^{S_{0}^{N \times \mathbb{R}}}$, and therefore the corresponding Dirac operator, does not preserve the decomposition $S_{0}^{N \times \mathbb{R}}=S_{+}^{N \times \mathbb{R}} \oplus S_{-}^{N \times \mathbb{R}}$. With respect to this decomposition, that Dirac operator has the form

$$
\left(\begin{array}{cc}
D_{0}^{S_{+}^{N \times \mathbb{R}}} & A \\
B & D_{0}^{S_{-}^{N \times \mathbb{R}}}
\end{array}\right)
$$

for vector bundle homomorphisms $A: S_{-}^{N \times \mathbb{R}} \rightarrow S_{+}^{N \times \mathbb{R}}$ and $B: S_{+}^{N \times \mathbb{R}} \rightarrow$ $S_{-}^{N \times \mathbb{R}}$. (See Section 5.16 in [6], or use the fact that the difference of two connections is an endomorphism-valued one-form.) The Dirac operator $D_{0}^{C}$ equals this operator on $N \times(1 / 4, \infty)$. Let $\nabla_{ \pm}^{S_{ \pm}^{N \times \mathbb{R}}}$ be the pullback of $\nabla^{S_{ \pm}^{N}}$ to a connection on $S_{ \pm}^{N \times \mathbb{R}}$. Consider a Clifford connection $\nabla^{S_{0}^{C}}$ on $S_{0}^{C}$ that is equal to the direct sum of $\nabla^{S_{+}^{N \times \mathbb{R}}}$ and $\nabla^{S_{-}^{N \times \mathbb{R}}}$ on $N \times(1 / 2, \infty)$ and to $\nabla^{S_{0}}$ on $M_{-} \backslash U$. Then the corresponding Dirac operator $\tilde{D}_{0}^{C}$ is equal to

$$
\left(\begin{array}{cc}
D_{0}^{S_{+}^{N \times \mathbb{R}}} & 0 \\
0 & D_{0}^{S_{-}^{N \times \mathbb{R}}}
\end{array}\right)
$$

on $N \times(1 / 2, \infty)$ and to $D_{0}$ on $M_{-} \backslash U$.

Lemma 5.2. There exists a $\lambda \geq 1$ such that $\lambda \Phi^{C}$ is admissible for $\tilde{D}_{0}^{C}$. For such $\lambda$,

$$
\operatorname{index}_{G}\left(D^{C}+\Phi^{C}\right)=\operatorname{index}_{G}\left(\tilde{D}^{C}+\lambda \Phi^{C}\right) .
$$

Proof. Existence of $\lambda$ with the desired property can be established as in the proof of Lemma 5.13 in [9]. The equality (5.4) can be proved via a linear homotopy; again see the proof of Lemma 5.13 in [9] for details, where references to Proposition 4.1 in [9] should be replaced by references to Proposition 4.9 
in the current paper, and one uses Propositions 4.3 and 4.4 to check that the first condition in Proposition 4.9 is satisfied.

To be explicit, an application of Proposition 4.9 shows that

$$
\operatorname{index}_{G}\left(D^{C}+\lambda \Phi^{C}\right)=\operatorname{index}_{G}\left(\tilde{D}^{C}+\lambda \Phi^{C}\right) .
$$

This follows from Proposition 4.9 by setting $P=D^{C}+\lambda \Phi^{C}, \Psi=\left(\begin{array}{cc}0 & A \\ B & 0,\end{array}\right)$, with $A$ and $B$ as in (5.3), $t_{0}=-1$ and $t_{1}=0$. Note that although $\Psi$ is not a Callias-type potential here, Proposition 4.9 still applies, since $\Psi$ defines an element of $\mathcal{L}\left(\mathcal{E}^{0}\right)$ by Proposition 4.3 and an element of $\mathcal{L}\left(\mathcal{E}^{1}\right)$ by Proposition 4.4. (The conditions of Proposition 4.4 hold because of the forms of $D^{C}$, $\Phi^{C}$ and $\Psi$.) A more straightforward application of Proposition 4.9 yields $\operatorname{index}_{G}\left(D^{C}+\Phi^{C}\right)=\operatorname{index}_{G}\left(D^{C}+\lambda \Phi^{C}\right)$.

Let $\chi \in C^{\infty}(\mathbb{R})$ be an odd function such that

$$
\chi(t)= \begin{cases}0 & \text { if }-1 / 4 \leq t \leq 1 / 4 \\ 1 & \text { if } 3 / 4 \leq t \leq 3 / 2 \\ t & \text { if } t \geq 2\end{cases}
$$

(The property that $\chi$ is unbounded in a way that makes it proper is only used in the proof of Lemma 5.6, see Lemma 5.5.) Such functions form a subset of the set of functions $\chi$ in Subsection 5.1. but Proposition 5.1 for general $\chi$ follows from the case for this class of functions, because the index on the right-hand side of (5.1) does not change if we modify $\chi$ in a cocompact set. (And at any rate, to prove Theorem 3.4, we only need Proposition 5.1 to hold for one such function $\chi$.)

Let $\gamma^{N}$ be the grading operator on $\left.S_{0}\right|_{N}$ that equals \pm 1 on $S_{ \pm}^{N}$. Let $\gamma^{N \times \mathbb{R}}$ be its pullback to $S_{0}^{N \times \mathbb{R}}$. Let $\Phi_{0}^{\chi}$ be the endomorphism of $S_{0}^{C}$ equal to $\chi \gamma^{N \times \mathbb{R}}$ on $N \times(1 / 4, \infty)$ and equal to zero on the rest of $M_{C}$.

Lemma 5.3. We have

$$
\operatorname{index}_{G}\left(\tilde{D}^{C}+\lambda \Phi^{C}\right)=\operatorname{index}_{G}\left(\tilde{D}^{C}+\Phi^{\chi}\right) .
$$

Proof. This can be proved via a linear homotopy between $\lambda \Phi^{C}$ and $\Phi^{\chi}$. The details are precisely as in the proof of Lemma 5.15 in [9], with references to Propositions 4.1 and 5.9 in [9] replaced by references to Proposition 4.9 (combined with Propositions 4.3 and 4.4) and Corollary 4.14, respectively, in the present paper.

5.3. Proof of Proposition 5.1. Let $M_{-}^{-}$be the manifold $M_{-}$with reversed orientation. Form the manifold

$$
M_{C}^{-}:=(N \times(-\infty, 1]) \cup_{N} M_{-}^{-} .
$$

See Figure 4. (The notation is motivated by the fact that $M_{C}$ with reversed orientation is naturally equal to $(N \times(-\infty,-1]) \cup_{N} M_{-}^{-}$, which can be identified with $M_{C}^{-}$via a shift over a distance 2 .) 


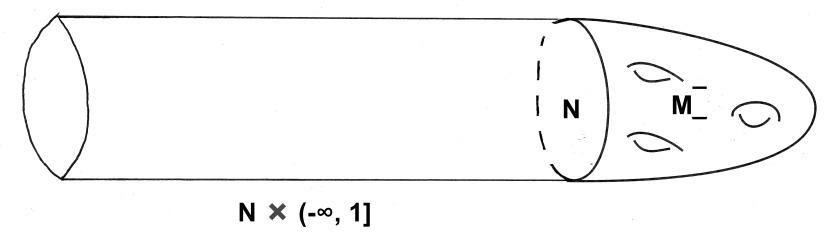

Figure 4. The manifold $M_{C}^{-}$

Let $S_{0}^{-} \rightarrow M_{-}^{-}$be equal to the vector bundle $\left.S_{0}\right|_{M_{-}}$, but with the opposite Clifford action (where $v \in T M_{-}^{-}$acts as $c(-v)$ ). Let $S_{0}^{C,-} \rightarrow M_{C}^{-}$be the Clifford module that is equal to $S_{0}^{N \times \mathbb{R}}$ on $N \times(-\infty, 5 / 4]$ and to $S_{0}^{-}$on $M_{-}^{-}$. From the Clifford connections $\nabla_{ \pm}^{S_{ \pm}^{N \times \mathbb{R}}}$ on $S_{ \pm}^{N \times \mathbb{R}}$ and a Clifford connection $\nabla^{S_{0}^{-}}$on $S_{0}^{-}$, construct a Clifford connection $\nabla^{S_{0}^{C,-}}$ on $S_{0}^{C,-}$ by

$$
\nabla^{S_{0}^{C,-}}:= \begin{cases}\nabla_{+}^{S_{+}^{N \times \mathbb{R}}} \oplus \nabla^{S_{-}^{N \times \mathbb{R}}} & \text { on } N \times(-\infty, 5 / 4) ; \\ \nabla^{S_{0}^{-}} & \text {on } M_{-}^{-} .\end{cases}
$$

Using this connection, we obtain the Dirac operator $D_{0}^{C,-}$ on $\Gamma^{\infty}\left(S_{0}^{C,-}\right)$. Then $D_{0}^{C,-}$ equals $\tilde{D}_{0}^{C}$ on $N \times(1 / 2,5 / 4)$.

The function $\chi$ equals 1 on $(3 / 4,5 / 4)$. Thus on this interval, both $\Phi_{0}^{\chi}$ and $\left(\begin{array}{cc}\chi & 0 \\ 0 & -1\end{array}\right)$ are equal to $\gamma^{N \times \mathbb{R}}$. (Here we use $2 \times 2$ matrix notation with respect to the decomposition $S_{0}^{N \times \mathbb{R}}=S_{+}^{N \times \mathbb{R}} \oplus S_{-}^{N \times \mathbb{R}}$.) So we can define the endomorphism $\Phi_{0}^{C,-}$ of $S_{0}^{C,-}$ by setting it equal to $\Phi_{0}^{\chi}$ on $(N \times(3 / 4,1]) \cup_{N} M_{-}^{-}$ and equal to

$$
\left(\begin{array}{cc}
\chi & 0 \\
0 & -1
\end{array}\right)
$$

on $N \times(-\infty, 5 / 4)$, where $S_{0}^{C,-}=S_{0}^{N \times \mathbb{R}}=S_{+}^{N \times \mathbb{R}} \oplus S_{-}^{N \times \mathbb{R}}$.

Lemma 5.4. We have

$$
\operatorname{index}_{G}\left(D^{C,-}+\Phi^{C,-}\right)=0 .
$$

Proof. Because $\chi=-1$ on $(-\infty,-3 / 4)$, we can define the endomorphism $\tilde{\Phi}_{0}^{C,-}$ of $S_{0}^{C,-}$ by setting it equal to $\Phi_{0}^{C,-}$ on $N \times(-\infty,-3 / 4)$ (where it equals (5.5) ) and equal to -1 on $(N \times(-1,1]) \cup_{N} M_{-}^{-}$. For that endomorphism, the estimate (3.1) holds on all of $M$. Therefore by Lemma 4.7.

$$
\operatorname{index}_{G}\left(D^{C,-}+\tilde{\Phi}^{C,-}\right)=0 .
$$

The claim now follows from Corollary 4.10

Proof of Proposition 5.1. Consider the cylinder $N \times \mathbb{R}$ as in Figure 1. The data $\left(M_{C}, S_{0}^{C}, \Phi_{0}^{\chi}\right)$ and $\left(M_{C}^{-}, S_{0}^{C,-}, \Phi_{0}^{C,-}\right)$ coincide in a neighbourhood of 


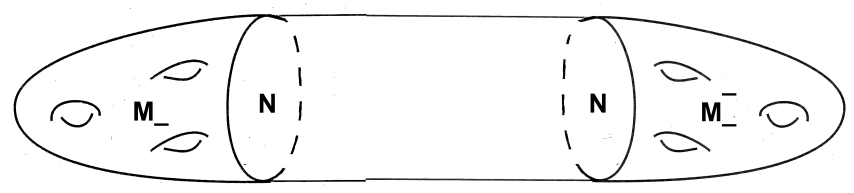

Figure 5. The manifold $M_{-} \cup_{N} M_{-}^{-}$

$N \times\{1\}$. By cutting along $N \times\{1\}$ and gluing, we obtain the corresponding data $\left(N \times \mathbb{R}, S_{0}^{N \times \mathbb{R}}, \Phi_{0}^{N \times \mathbb{R}}\right)$ and $\left(M_{-} \cup_{N} M_{-}^{-}, S_{0}^{M_{-} \cup_{N} M_{-}^{-}}, \Phi_{0}^{M_{-} \cup_{N} M_{-}^{-}}\right)$. See Figure 5. To be explicit,

$$
\Phi_{0}^{N \times \mathbb{R}}=\left(\begin{array}{cc}
\chi & 0 \\
0 & -1
\end{array}\right)
$$

on $S_{+}^{N \times \mathbb{R}} \oplus S_{-}^{N \times \mathbb{R}}$.

Theorem 4.13 implies that

$$
\begin{aligned}
\operatorname{index}_{G}\left(\tilde{D}^{C}+\Phi^{\chi}\right)+\operatorname{index}_{G}\left(D^{C,-}+\Phi^{C,-}\right) \\
\quad=\operatorname{index}_{G}\left(D^{N \times \mathbb{R}}+\Phi^{N \times \mathbb{R}}\right)+\operatorname{index}_{G}\left(D^{M_{-} \cup_{N} M_{-}^{-}}+\Phi^{M_{-} \cup_{N} M_{-}^{-}}\right) .
\end{aligned}
$$

By Lemmas 4.8 and 5.4 , this implies that

$$
\operatorname{index}_{G}\left(\tilde{D}^{C}+\Phi^{\chi}\right)=\operatorname{index}_{G}\left(D^{N \times \mathbb{R}}+\Phi^{N \times \mathbb{R}}\right) .
$$

The connection $\tilde{\nabla}^{S_{0}^{N \times \mathbb{R}}}$ on $S_{0}^{N \times \mathbb{R}}$ obtained from cutting and gluing the connections $\nabla^{S_{0}^{C}}$ and $\nabla^{S_{0}^{C,-}}$ is the direct sum connection $\nabla^{S_{+}^{N \times \mathbb{R}}} \oplus \nabla^{S_{+}^{N \times \mathbb{R}}}$. So the corresponding Dirac operator $D_{0}^{S_{0}^{N \times \mathbb{R}}}$ equals

$$
D_{0}^{S_{0}^{N \times \mathbb{R}}}=\left(\begin{array}{cc}
D_{0}^{S_{+}^{N \times \mathbb{R}}} & 0 \\
0 & D_{0}^{S_{+}^{N \times \mathbb{R}}}
\end{array}\right) .
$$

By the explicit form (5.6) of $\Phi_{0}^{N \times \mathbb{R}}$, the operator $D_{0}^{S_{0}^{N \times \mathbb{R}}} \pm i \Phi_{0}^{N \times \mathbb{R}}$ on $\Gamma^{\infty}\left(S_{0}^{N \times \mathbb{R}}\right)$ is the direct sum of the operators $D_{0}^{S_{+}^{N \times \mathbb{R}}} \pm i \chi$ on $\Gamma^{\infty}\left(S_{+}^{N \times \mathbb{R}}\right)$ and $D_{0}^{S_{-}^{N \times \mathbb{R}} \mp i}$ on $\Gamma^{\infty}\left(S_{-}^{N \times \mathbb{R}}\right)$. So $\operatorname{index}_{G}\left(D^{N \times \mathbb{R}}+\Phi^{N \times \mathbb{R}}\right)=\operatorname{index}_{G}\left(D^{S_{+}^{N \times \mathbb{R}}}+\chi^{N \times \mathbb{R}}\right)+\operatorname{index}_{G}\left(D^{S_{-}^{N \times \mathbb{R}}}+\left(\begin{array}{cc}0 & -i \\ i & 0\end{array}\right)\right)$.

Lemma 4.7 then implies that the second term on the right-hand side is zero, whence

$$
\operatorname{index}_{G}\left(\tilde{D}^{C}+\Phi^{\chi}\right)=\operatorname{index}_{G}\left(D^{S_{+}^{N \times \mathbb{R}}}+\chi^{N \times \mathbb{R}}\right)
$$


The claim now follows from $(5.2)$ in conjunction with Lemmas 5.2 and 5.3. As pointed out above Lemma 5.3, the case for the class of functions $\chi$ we have used implies the case of the more general functions $\chi$ allowed in Proposition 5.1.

5.4. Proof of Theorem 3.4. Let $\mathcal{E}_{N \times \mathbb{R}}$ be the Hilbert $C^{*}(G)$-module constructed from $\Gamma_{c}\left(S_{+}^{N \times \mathbb{R}}\right)$ as in Subsection 3.1. We write $D_{\chi}:=D_{+}^{S_{+}^{N \times \mathbb{R}}}+\chi^{N \times \mathbb{R}}$ for brevity.

Lemma 5.5. For all $a>0$ the operator $\left(D_{\chi}^{2}+a\right)^{-1}$ on $\mathcal{E}_{N \times \mathbb{R}}$ is compact.

Proof. This is (a special case of) an analogue of Theorem 2.40 in [11. The proof proceeds in the same way, with the difference that the operator $D_{\chi}^{2}$ can only be bounded below by a function $h$ that is $G$-proper, in the sense that the inverse image of a compact set is cocompact instead of compact. One chooses the bump functions $g_{n}$ in the proof of Theorem 2.40 in [11] to be $G$-invariant and cocompactly supported. Theorem 4.2, and Lemma 4.6(a) in [15], imply that $g_{n}\left(D_{\chi}^{2}+a\right)^{-1}$ is a compact operator on $\mathcal{E}_{N \times \mathbb{R}}$. And as in the proof of Theorem 2.40 in [11], one shows that $g_{n}\left(D_{\chi}^{2}+a\right)^{-1}$ converges to $\left(D_{\chi}^{2}+a\right)^{-1}$ in the operator norm on $\mathcal{L}\left(\mathcal{E}_{N \times \mathbb{R}}\right)$.

We will use an analogue of Theorem 6.6 in [9].

Lemma 5.6. The operator

$$
D_{\chi}\left(D_{\chi}^{2}+f\right)^{-1 / 2}-D_{\chi}\left(D_{\chi}^{2}+1\right)^{-1 / 2}
$$

lies in $\mathcal{K}\left(\mathcal{E}_{N \times \mathbb{R}}\right)$.

Proof. By Proposition 4.12 in [15], and as in (6.6) in [9], the operator (5.7) equals

$$
\frac{2}{\pi} \int_{0}^{\infty} D_{\chi}\left(D_{\chi}^{2}+f+\lambda^{2}\right)^{-1}(f-1)\left(D_{\chi}^{2}+1+\lambda^{2}\right)^{-1} d \lambda
$$

The operator $\left(D_{\chi}^{2}+1+\lambda^{2}\right)^{-1}$ is compact by Lemma 5.5. Lemmas 4.6 and 4.8 in [15] imply that the integrand in (5.8) is bounded by $a\left(b+\lambda^{2}\right)^{-1}$ for certain $a, b>0$, so the integral converges in operator norm. It therefore defines a compact operator.

Theorem 3.4 follows from Proposition 5.1 and the following fact.

Proposition 5.7. In the setting of Proposition 5.1,

$$
\operatorname{index}_{G}\left(D_{\chi}\right)=\operatorname{index}_{G}\left(D^{S_{+}^{N}}\right) .
$$

Proof. Let $\mathcal{E}_{N}$ be the Hilbert $C^{*}(G)$-module constructed from $\Gamma_{c}\left(S_{+}^{N}\right)$ as in Subsection 3.1. Then $\mathcal{E}_{N \times \mathbb{R}}=\mathcal{E}_{N} \otimes L^{2}(\mathbb{R})$. So Lemma 5.6 implies that $\operatorname{index}_{G}\left(D_{\chi}\right)$ is represented by the unbounded Kasparov cycle

$$
\left(\mathcal{E}_{N} \otimes L^{2}(\mathbb{R}) \otimes \mathbb{C}^{2}, D_{\chi}\right)
$$


The Callias-type operator $D_{\chi}$ on

$$
\Gamma^{\infty}\left(S_{+}^{N \times \mathbb{R}} \oplus S_{+}^{N \times \mathbb{R}}\right)=\Gamma^{\infty}\left(S_{+}^{N}\right) \otimes C^{\infty}(\mathbb{R}) \otimes \mathbb{C}^{2}
$$

is equal to

$$
\left(\begin{array}{cc}
0 & D^{S_{+}^{N}} \\
D^{S_{+}^{N}} & 0
\end{array}\right) \otimes 1_{C^{\infty}(\mathbb{R})}+\gamma_{S_{+}^{N}} \otimes\left(\begin{array}{cc}
0 & i \frac{d}{d t} \\
i \frac{d}{d t} & 0
\end{array}\right)+1_{\Gamma_{c}^{\infty}\left(S_{+}^{N}\right)} \otimes\left(\begin{array}{cc}
0 & -i \chi \\
i \chi & 0
\end{array}\right)
$$

where $\gamma_{S_{+}^{N}}$ is the grading operator on $S_{+}^{N}$, equal to $-i$ times Clifford multiplication by the unit normal vector field $\hat{n}$ on $N$ pointing into $M_{+}$(so that $\left.\gamma_{S_{+}^{N}} \otimes i \frac{d}{d t}=c(\hat{n}) \otimes \frac{d}{d t}\right)$. Let $\mathcal{E}_{N}^{ \pm}$be the even and odd-graded parts of $\mathcal{E}_{N}$, and let $D_{ \pm}^{S_{+}^{N}}$ be the restriction of $D^{S_{+}^{N}}$ to even and odd-graded sections of $S_{+}^{N}$, respectively. With respect to the decomposition

$\mathcal{E}_{N} \otimes L^{2}(\mathbb{R}) \otimes \mathbb{C}^{2}=\left(\mathcal{E}_{N}^{+} \otimes L^{2}(\mathbb{R})\right) \oplus\left(\mathcal{E}_{N}^{-} \otimes L^{2}(\mathbb{R})\right) \oplus\left(\mathcal{E}_{N}^{+} \otimes L^{2}(\mathbb{R})\right) \oplus\left(\mathcal{E}_{N}^{-} \otimes L^{2}(\mathbb{R})\right)$,

the operator 5.10 ) equals

$$
D_{\chi}=\left(\begin{array}{cccc}
0 & 0 & 1_{\mathcal{E}_{N}^{+}} \otimes\left(i \frac{d}{d t}-i \chi\right) & D_{-}^{S_{+}^{N}} \otimes 1_{L^{2}(\mathbb{R})} \\
0 & 0 & D_{+}^{S_{+}^{N}} \otimes 1_{L^{2}(\mathbb{R})} & 1_{\mathcal{E}_{N}^{-}} \otimes\left(-i \frac{d}{d t}-i \chi\right) \\
1_{\mathcal{E}_{N}^{+} \otimes\left(i \frac{d}{d t}+i \chi\right)} & D_{-}^{S_{+}^{N}} \otimes 1_{L^{2}(\mathbb{R})} & 0 & 0 \\
D_{+}^{S_{+}^{N}} \otimes 1_{L^{2}(\mathbb{R})} & 1_{\mathcal{E}_{N}^{-}} \otimes\left(-i \frac{d}{d t}+i \chi\right) & 0 & 0
\end{array}\right)
$$

The kernel of $i \frac{d}{d t} \pm i \chi$ in $C^{\infty}(\mathbb{R})$ is one-dimensional, and spanned by the function

$$
f_{ \pm}(t)=e^{\mp \int_{0}^{t} \chi(u) d u}
$$

By the properties of $\chi, f_{+} \in L^{2}(\mathbb{R})$, whereas $f_{-} \notin L^{2}(\mathbb{R})$. It follows that $i \frac{d}{d t}-i \chi$ is invertible on the appropriate domain, while $i \frac{d}{d t}+i \chi$ is zero on $\mathbb{C} f_{+}$and invertible on $f_{+}^{\perp}$.

Consider the submodules

$$
\begin{aligned}
& \mathcal{E}_{1}:=\left(\mathcal{E}_{N}^{+} \otimes \mathbb{C} f_{+}\right) \oplus 0 \oplus 0 \oplus\left(\mathcal{E}_{N}^{-} \otimes \mathbb{C} f_{+}\right) ; \\
& \mathcal{E}_{2}:=\mathcal{E}_{1}^{\perp}=\left(\mathcal{E}_{N}^{+} \otimes f_{+}^{\perp}\right) \oplus\left(\mathcal{E}_{N}^{-} \otimes L^{2}(\mathbb{R})\right) \oplus\left(\mathcal{E}_{N}^{+} \otimes L^{2}(\mathbb{R})\right) \oplus\left(\mathcal{E}_{N}^{-} \otimes f_{+}^{\perp}\right)
\end{aligned}
$$

of (5.11). These are preserved by the operator $D_{\chi}$. (For $\mathcal{E}_{1}$, this is immediate from (5.12); for $\mathcal{E}_{2}$, this follows from the facts that $D_{\chi}$ is symmetric and preserves $\mathcal{E}_{1}$.) We find that the cycle $(5.9)$ decomposes as

$$
\left(\mathcal{E}_{1},\left(\begin{array}{cccc}
0 & 0 & 0 & D_{-}^{S_{+}^{N}} \otimes 1_{\mathbb{C} f_{+}} \\
0 & 0 & 0 & 0 \\
0 & 0 & 0 & 0 \\
D_{+}^{S_{+}^{N}} \otimes 1_{\mathbb{C} f_{+}} & 0 & 0 & 0
\end{array}\right)\right) \oplus\left(\mathcal{E}_{2}, D_{\chi} \mid \mathcal{E}_{2}\right) .
$$

The operator $\left.D_{\chi}\right|_{\mathcal{E}_{2}}$ is essentially self-adjoint on the initial domain of compactly supported smooth sections by Proposition 5.5 in [15], and its 
square has a positive lower bound in the Hilbert $C^{*}(G)$-module sense. Thus its self-adjoint closure is invertible, so that the second term in (5.13) is homotopic to a degenerate cycle. The first term represents $\operatorname{index}_{G}\left(D^{S_{+}^{N}}\right)$.

Remark 5.8. A similar argument in the case where $G$ is trivial is hinted at below Lemma 4.1 in [25].

\section{Proofs of Results on Positive scalar CURVAture}

6.1. Obstruction results. We now deduce Theorem 2.1 from Theorem 3.4, and Corollary 2.4 from Theorem 2.1 and index theorems in [24, 40, 41].

Proof of Theorem 2.1. This proof is an adaptation of the proof of Theorem 2.1 in [2].

First suppose that $M$ is odd-dimensional. Let $\kappa$ denote scalar curvature. Let $K \subset \bar{X}$ be a cocompact subset of $M$ such that $N \subset K, \kappa>0$ on $K$, and the distance from $X \backslash K$ to $Y$ is positive. Let $\chi \in C^{\infty}(M)^{G}$ be a function such that $\chi(x)=1$ for all $x \in Y$ and $\chi(x)=-1$ for all $x \in X \backslash K$. Consider the operator $D$ as in (3.5), where $D_{0}$ is the Spin-Dirac operator on $M$, and the admissible endomorphism $\Phi$ as in (3.6), where $\Phi_{0}$ is pointwise multiplication by $\chi$. Then the set $M_{-}$in Subsection 3.2 can be chosen to be cocompact as required, and so that $N=N^{-} \cup H$, where $\left.f\right|_{N^{-}}=-1$. In this setting,

$$
S_{+}^{N}=\left.S_{0}\right|_{H}
$$

where $S_{0} \rightarrow M$ is the spinor bundle. (This is consistent with Corollary 7.3.)

For any $\lambda \in \mathbb{R}$, Lichnerowicz' formula implies that

$$
\left(D_{0} \pm i \lambda \chi\right)^{2}=D_{0}^{2} \pm i \lambda c(d \chi)+\lambda^{2} \chi^{2} \geq \kappa / 4-\lambda\|d \chi\|+\lambda^{2} \chi^{2} .
$$

On $M \backslash K$, the function on the right-hand side equals $\kappa / 4+\lambda^{2} \geq \lambda^{2}$. Since $\kappa$ is $G$-invariant, and positive on the cocompact set $K$, it has a positive lower bound on that set. Further, $d \chi$ is $G$-invariant and cocompactly supported, hence bounded. So we can choose $\lambda>0$ small enough so that $\kappa / 4-\lambda\|d \chi\|>$ 0 on $K$. It follows that $\left(D_{0} \pm i \lambda \chi\right)^{2}$ has a positive lower bound. This implies that $D+\Phi$ is invertible, so $\operatorname{index}_{G}(D+\Phi)=0$. The claim then follows by Theorem 3.4 and 6.1).

If $M$ is even-dimensional, then the claim follows by applying the result in the odd-dimensional case to the manifold $M \times S^{1}$.

Proof of Corollary 2.4. This follows from Theorem 2.1 and index formulas for traces defined by orbital integrals applied to $\operatorname{index}_{G}\left(D^{H}\right)$. These index formulas are:

- Theorem 6.10 in [41] if $G$ is any locally compact group and $g=e$;

- Theorem 6.1 in [40] if $G$ is discrete and finitely generated and $g$ is any element; 
- Proposition 4.11 in [24] if $G$ is a connected semisimple Lie group and $g$ is a semisimple element.

These results imply that in the setting of Corollary 2.4.

$$
\tau_{g}\left(\operatorname{index}_{G}\left(D^{H}\right)\right)=\hat{A}_{g}(H),
$$

for a trace $\tau_{g}$. As index ${ }_{G}\left(D^{H}\right)$ is independent of the choice of Riemannian metric, so is $\hat{A}_{g}(H)$. Finally, vanishing of $\operatorname{index}_{G}\left(D^{H}\right)$ implies vanishing of $\hat{A}_{g}(H)$ for all $g$ as above.

6.2. Existence result. In the remainder of this section, we prove Theorem 2.9 by generalising a construction by Lawson and Yau [27]. We first prove in this subsection an extension of Theorem 3.8 in [27], namely Proposition 6.1 ,

To prepare, let us recall the steps in the construction of Lawson and Yau's positive scalar curvature metrics [27], which we denote by $\tilde{g}_{t}$, on a compact manifold $N$.

Let $K$ be a compact Lie group acting on $N$. Consider the principal $K$ bundle defined by the map

$$
p: K \times N \rightarrow N, \quad(k, y) \mapsto k^{-1} y .
$$

Take a $K$-invariant Riemannian metric $g_{N}$ on $N$. Let $b$ be a bi-invariant Riemannian metric on $K$. Let $\hat{g}$ denote the lift of $g_{N}$ to the orthogonal complement to $\operatorname{ker}(T p)$ in $T(K \times N)$ with respect to the product metric $b \oplus g_{N}$ on $K \times N$.

For each $t>0$, let $\hat{b}_{t^{2}}$ be the lift of the metric $t^{2} b$ on $K$ to $T K \times N \subset$ $T(K \times N)$. Then

$$
g_{t}:=\hat{g} \oplus \hat{b}_{t^{2}}
$$

is a Riemannian metric on the total space $K \times N$. One can check that, for each $t, g_{t}$ is invariant under the left $K$-action on $K \times N$ defined by

$$
l \cdot(k, y)=\left(k l^{-1}, y\right) .
$$

Thus $g_{t}$ descends, via the projection onto the second factor,

$$
\pi: K \times N \rightarrow N, \quad(k, y) \mapsto y,
$$

to a $K$-invariant metric $\tilde{g}_{t}$ on $N$. Further, one sees that $\pi$ is a Riemannian submersion with respect to the metrics $g_{t}$ and $\tilde{g}_{t}$ on the total space and base respectively.

The following proposition shows that, under the conditions stated, for all sufficiently small $t, \tilde{g}_{t}$ has positive scalar curvature outside a neighbourhood of the fixed point set. It is an adaptation of the proof of Theorem 3.8 in [27] to the more general setting when $N$ is non-compact but has $K$-bounded geometry; see Definition 2.8 .

Proposition 6.1. Let $N$ be a manifold with an action by a non-abelian, compact, connected Lie group $K$. Fix a bi-invariant metric on $K$. If $g_{N}$ is a K-invariant Riemannian metric on $N$ with $K$-bounded geometry, then for 
any neighbourhood $U$ of the fixed point set $N^{K}$, there exists $t_{U}>0$ such that for all $t \leq t_{U}$, the metric $\tilde{g}_{t}$ constructed above has uniform positive scalar curvature on $N \backslash U$.

Proof. We will follow the steps in the proof of Theorem 3.8 in [27] and show where the assumptions of bounded geometry and no shrinking orbits (Definition 2.5) are needed to obtain the conclusion.

For each $y \in N \backslash N^{K}$, there is an orthogonal splitting $\mathfrak{k}=\mathfrak{k}_{y} \oplus \mathfrak{p}_{y}$, where $\mathfrak{k}_{y}$ is the Lie subalgebra of the isotropy subgroup $K_{y}$ of $y$. The map $\varphi_{y}$ from (2.1) restricts to an injection on $\mathfrak{p}_{y}$. Denote the orthogonal complement of $\varphi_{y}\left(\mathfrak{p}_{y}\right)$ in $T_{y} N$ by $V_{y}$. Then

$$
T_{(e, y)}(K \times N) \cong \mathfrak{k}_{y} \oplus \mathfrak{p}_{y} \oplus \varphi_{y}\left(\mathfrak{p}_{y}\right) \oplus V_{y} .
$$

For each $y \in N \backslash N^{K}$, choose an orthonormal basis $\left\{e_{1}(y), \ldots, e_{l_{y}}(y)\right\}$ of $\mathfrak{p}_{y}$ with respect to $b$ such that for all $j, k=1, \ldots, l_{y}$,

$$
g\left(\varphi_{y}\left(e_{j}(y)\right), \varphi_{y}\left(e_{k}(y)\right)\right)=\sigma_{j}^{2}(y) \delta_{j k}
$$

for some continuous, positive functions $\sigma_{j}$. For each $j=1, \ldots, l_{y}$, define a function $\lambda_{j}: N \backslash N^{K} \rightarrow(0, \infty)$ by

$$
\lambda_{j}(y):=\sigma_{j}(y)\left(1+\sigma_{j}(y)^{2}\right) .
$$

By the calculations in the proofs of Propositions 3.6 and 3.7 in [27] and the assumption that $g_{N}$ has bounded geometry, for any neighbourhood $U$ of $N^{K}$, the scalar curvature of $\tilde{g}_{t}$ at any $y \in N \backslash U$ is bounded below by

$$
\sum_{j, k=1}^{l_{y}} \frac{1}{t^{2}} \frac{\lambda_{j}(y)^{2} \lambda_{k}(y)^{2}}{\left(t^{2}+\lambda_{j}(y)^{2}\right)\left(t^{2}+\lambda_{k}(y)^{2}\right)}\left\|\left[e_{j}(y), e_{k}(y)\right]\right\|_{b}^{2}+O(1)
$$

as $t \rightarrow 0$, where the $O(1)$ term is independent of $y$. Since the $K$-action has no shrinking orbits with respect to $g_{N}$, there exists $c_{U}>0$ such that $\lambda_{j}(y)>c_{U}$ for each $y \in N \backslash U$ and $j=1, \ldots, l_{y}$. In particular, for $t \leq c_{U}$, the expression 6.2 is bounded below by

$$
\sum_{j, k=1}^{l_{y}} \frac{1}{4 t^{2}}\left\|\left[e_{j}(y), e_{k}(y)\right]\right\|_{b}^{2}+O(1)
$$

Now, without loss of generality we may assume that $K=\mathrm{SU}(2)$ or $K=$ $\mathrm{SO}(3)$, as any compact, connected, non-abelian Lie group has such a subgroup. Since $K$ has no subgroups of codimension 1 , we have $l_{y}=\operatorname{dim} \mathfrak{p}_{y} \geq 2$ at each $y \in N \backslash N^{K}$. For all $j$ and $k,\left\|\left[e_{j}(y), e_{k}(y)\right]\right\|_{b}^{2}$ is 4 times the sectional curvature of the plane spanned by $e_{j}(y)$ and $e_{k}(y)$ with respect to the metric $b$, and this is constant in $y$, and positive for $K=\mathrm{SU}(2)$ or $K=\mathrm{SO}(3)$. Thus for any neighbourhood $U$ of $N^{K}$, there exists $t_{U}>0$ such that for all $t \leq t_{U}$, the expression (6.3), and hence also (6.2), is uniformly positive outside $U$. It follows that for all such $t$, the scalar curvature of $\tilde{g}_{t}$ is uniformly positive outside $U$. 
We now deduce Theorem 2.9 from the following noncompact generalisation of the main result in [27].

Theorem 6.2. Let $N$ be a manifold that admits an effective action by a compact, connected, non-abelian Lie group $K$, such that the fixed point set $N^{K}$ is compact. If there exists a $K$-invariant Riemannian metric on $N$ such that the $K$-action has $K$-bounded geometry, then $N$ admits a $K$-invariant metric with uniformly positive scalar curvature.

Proof. Since $N^{K}$ is compact and the action is effective, by Section 4 of 27. there exists $t_{0}>0$, a $K$-invariant neighbourhood $U$ of $K$ with compact closure, and a $K$-invariant Riemannian metric $g^{\prime}$ on $N$ such that each metric $\tilde{g}_{t}^{\prime}$, constructed from $g^{\prime}$ as in subsection 6.2, has positive scalar curvature on $U$ for $0<t<t_{0}$.

Fix a bi-invariant metric $b$ on $K$. Let $g^{\prime \prime}$ be a $K$-invariant metric on $N$ for which the $K$-action has $K$-bounded geometry. Let $\left\{f_{1}, f_{2}\right\}$ be a smooth, $K$-invariant partition of unity on $N$ such that $f_{1} \equiv 1$ on $U$ and $f_{1} \equiv 0$ on $N \backslash U^{\prime}$, where $U^{\prime}$ is a relatively compact neighbourhood of $N^{K}$ containing the closure of $U$. Then

$$
g_{N}:=f_{1} g^{\prime}+f_{2} g^{\prime \prime}
$$

is a $K$-invariant Riemannian metric on $N$. Applying the prescription in Subsection 6.2 to $g_{N}$, we obtain a family $\left\{\tilde{g}_{t}\right\}_{t>0}$ of $K$-invariant metrics on $N$. We claim that for sufficiently small $t, \tilde{g}_{t}$ has uniformly positive scalar curvature on $N$.

To see this, let $\|\varphi\|$ be the norm function $(2.2)$ associated to the metric $g_{N}$. Since $g^{\prime \prime}$ and $g_{N}$ coincide on $N \backslash U^{\prime}$, and $g^{\prime \prime}$ has $K$-bounded geometry, there exists $C_{U^{\prime}}>0$ such that $\|\varphi\|(y) \geq C_{U^{\prime}}$ for all $y \in N \backslash U^{\prime}$. One sees that $g_{N}$ has $K$-bounded geometry, and so by Proposition 6.1, there exists $t_{1}=\left(t_{1}\right)_{U}>0$ such that for all $t \leq t_{1}, \tilde{g}_{t}$ has uniformly positive scalar curvature on $N \backslash U$. It follows that for all $t \leq \min \left\{t_{0}, t_{1}\right\}, \tilde{g}_{t}$ has uniformly positive scalar curvature on $N$.

Proof of Theorem 2.9. In the setting of Theorem 2.9, Theorem 6.2 implies that $N$ admits a $K$-invariant metric with uniformly positive scalar curvature. By Theorem 4.6 in [17] (see also Theorem 58 in [18]), this metric induces a $G$-invariant Riemannian metric on $G \times_{K} N$ of uniformly positive scalar curvature.

\section{Further applications of the Callias-type indeX theOREM}

We used Theorem 3.4 to prove Theorem 2.1 in Subsection 6.1. We give some other applications of Theorem 3.4 here.

7.1. The image of the assembly map. If $M / G$ is noncompact, and $G$ is not known to satisfy Baum-Connes surjectivity, then it is a priori unclear if index $\operatorname{dex}_{G}(D+\Phi)$ lies in the image of the Baum-Connes assembly map [4]; see the question raised on page 3 of [15. Theorem 3.4 implies that this is in fact the case for $G$-Callias-type operators as defined above: 
Corollary 7.1. The Callias-index of $D+\Phi$ lies in the image of the BaumConnes assembly map.

7.2. Cobordism invariance of the assembly map. Theorem 3.4 leads to a perspective on cobordism invariance of the analytic assembly map.

Corollary 7.2. Let $X$ be an odd-dimensional Riemannian manifold with boundary $N$, on which $G$ acts properly and isometrically, preserving $N$, such that $M / G$ is compact. Suppose that a neighbourhood $U$ of $N$ is $G$ equivariantly isometric to $N \times[0, \varepsilon)$, for some $\varepsilon>0$. Let $S_{0}^{X} \rightarrow X$ be a $G$-equivariant Clifford module, and consider the Clifford module $\left.S_{0}^{X}\right|_{N} \rightarrow N$, graded by $i$ times Clifford multiplication by the inward-pointing unit normal. Suppose that $\left.\left.S_{0}^{X}\right|_{U} \cong S_{0}^{X}\right|_{N} \times[0, \varepsilon)$. Let $D^{N}$ be the Dirac operator on $\left.S_{0}^{X}\right|_{N}$ associated to a G-invariant Clifford connection $\nabla^{N}$ for the Clifford action by $T N$ on $\left.S_{0}^{X}\right|_{N}$. Then $\operatorname{index}_{G}\left(D^{N}\right)=0$.

Proof. Form the manifold $M$ by attaching the cylinder $N \times(0, \infty)$ to $X$ along $U$. Extend $S_{0}^{X}$ and the Clifford action to $M$ in the natural way. We write $S_{0}$ for the extension of $S_{0}^{X}$ to $M$. The connection $\nabla^{N}$ pulls back to a Clifford connection on $\left.S_{0}\right|_{N \times(0, \infty)}$. Because the $G$-invariant Clifford connections form an affine space, we can extend this pulled-back connection to all of $M$ using a $G$-invariant Clifford connection on $X \backslash N$ and a partition of unity. Let $D_{0}$ be the associated Dirac operator.

Let $\Phi_{0}$ be the identity endomorphism on $S_{0}$. Then $\Phi$, as in $(3.6)$ is admissible for $D$ as in (3.5), and (3.1) holds on all of $M$. By Lemma 4.7, this implies that $\operatorname{index}_{G}(D+\Phi)=0$. In this case, $S_{+}^{N}=\left.S_{0}^{X}\right|_{N}$, so by Theorem 3.4, $\operatorname{index}_{G}\left(D^{N}\right)=0$.

\subsection{Spin $^{c}$-Dirac operators.}

Corollary 7.3. Consider the setting of Theorem 3.4, and assume that $M$ is odd-dimensional and $D_{0}$ is a $\mathrm{Spin}^{c}$-Dirac operator. Then there is a union $N^{+}$of connected components of $N$ and a Spin ${ }^{c}$-structure on $N^{+}$with spinor bundle $\left.S_{0}\right|_{N^{+}}$such that

$$
\operatorname{index}_{G}(D+\Phi)=\operatorname{index}_{G}\left(D^{\left.S_{0}\right|_{N}+}\right) \quad \in K_{0}\left(C^{*}(G)\right),
$$

where $\left.D^{S_{0}}\right|_{N^{+}}$is a $\mathrm{Spin}^{c}$-Dirac operator on the spinor bundle $\left.S_{0}\right|_{N^{+}} \rightarrow N^{+}$. If $N$ is connected, then $\operatorname{index}_{G}(D+\Phi)=0$.

Proof. Since $S_{0}$ is an irreducible Clifford module, and $\left.S_{+}^{N} \subset S_{0}\right|_{N}$ is invariant under the Clifford action of $\left.T M\right|_{N}$, over each connected component $X$ of $N$ the bundle $\left.S_{+}^{N}\right|_{X}$ is either zero or $\left.S_{0}\right|_{X}$. Since $M$ is odd-dimensional, $\left.S_{0}\right|_{X}$ is the spinor bundle of the $\operatorname{Spin}^{c}$-structure of $X$ that it inherits from $M$. So (7.1) follows.

If $N$ is connected, then we either have $N^{+}=\emptyset$, in which case $\operatorname{index}_{G}(D+$ $\Phi)=0$ because $S_{+}^{N}$ is the zero bundle, or $N^{+}=N$, in which case (7.1) and 
Lemma 4.7 imply that

$$
\operatorname{index}_{G}(D+\Phi)=\operatorname{index}_{G}\left(D+\left(\begin{array}{cc}
0 & i \\
-i & 0
\end{array}\right)\right)=0 .
$$

There is a converse to Corollary 7.3 in the following sense. Let $N^{+} \subset N$ be any union of connected components; there is a finite number of such subsets of $N$ since $N / G$ is compact. We can define an admissible endomorphism $\Phi_{0}$ such that (7.1) holds, by taking $\Phi_{0}$ to be multiplication by a $G$-invariant function on $M$ that equals 1 on $N^{+}$and -1 on $N \backslash N^{+}$, and is constant 1 or -1 outside a cocompact set. Thus given any hypersurface $N$ bounding a cocompact set, and any set $N^{+}$of connected components of $N$, we have an index

$$
\operatorname{index} N_{G}^{N^{+}}(D):=\operatorname{index}_{G}(D+\Phi),
$$

with $\Phi$ and $\Phi_{0}$ related as in (3.6), independent of the choice of $\Phi_{0}$ with the property that $\Phi_{0}$ is positive definite on $N^{+}$and negative definite on $N \backslash N^{+}$.

Versions of the index $(7.2)$ are sometimes used in applications of Calliastype index theorems to obstructions to positive scalar curvature for Spinmanifolds, see [2, 9] and the proof of Theorem 2.1 in Subsection 6.1.

7.4. Induction. Suppose that $G$ is an almost connected, reductive Lie group, and let $K<G$ be maximal compact. In [18, 19, 24] some results were proved relating $G$-equivariant indices to $K$-equivariant indices via Dirac induction. Such results allow one to deduce results in equivariant index theory for actions by noncompact groups from corresponding results for compact groups. This was applied to obtain results in geometric quantisation [19, 20, 22] and geometry of group actions [18, 21]. Corollary 7.4 below is a version of this idea for the index of Definition 3.3.

To state this corollary, we consider the setting of Subsection 3.2. Using Abels' slice theorem, we write $M=G \times_{K} Y$, for a $K$-invariant submanifold $Y \subset M$, and $S_{0}=G \times\left._{K} S_{0}\right|_{Y}$. Let $\mathfrak{g}=\mathfrak{k} \oplus \mathfrak{p}$ be a Cartan decomposition. Then $T M \cong G \times_{K}(T Y \oplus \mathfrak{p})$. We assume that the $G$-invariant Riemannian metric on $M$ is induced by a $K$-invariant Riemannian metric on $Y$ and an $\operatorname{Ad}(K)$-invariant inner product on $\mathfrak{p}$ via this identification.

We assume for simplicity that the adjoint representation $\mathrm{Ad}: K \rightarrow \mathrm{SO}(\mathfrak{p})$ lifts to the double cover $\operatorname{Spin}(\mathfrak{p})$ of $\mathrm{SO}(\mathfrak{p})$. (This is true for a double cover of $G$.) Then the standard Spin representation $S_{\mathfrak{p}}$ of $\operatorname{Spin}(\mathfrak{p})$ may be viewed as a representation of $K$. We assume that $\left.S_{0}\right|_{Y}=S_{0}^{Y} \otimes S_{\mathfrak{p}}$ for a Clifford module $S_{0}^{Y} \rightarrow Y$. The Clifford action by $\left.T M\right|_{Y}$ on $\left.S_{0}\right|_{Y}$ equals $c_{Y} \otimes 1_{S_{\mathfrak{p}}}+1_{S_{0}^{Y}} \otimes c_{\mathfrak{p}}$, for a Clifford action $c_{Y}$ by $T Y$ on $S_{0}^{Y}$, and the Clifford action $c_{\mathfrak{p}}$ of $\mathfrak{p}$ on $S_{\mathfrak{p}}$. We choose the $G$-invariant connection $\nabla^{S_{0}}$ so that $\left.\nabla^{S_{0}}\right|_{Y}=\nabla^{S_{0}^{Y}} \otimes 1_{S_{\mathfrak{p}}}+$ $1_{S_{0}^{Y}} \otimes \nabla^{S_{\mathfrak{p}}}$, for Clifford connections $\nabla^{S_{0}^{Y}}$ on $S_{0}^{Y}$ and $\nabla^{S_{\mathfrak{p}}}$ on $Y \times S_{\mathfrak{p}}$

Since $\Phi_{0}$ is $G$-equivariant, it is determined by its restriction to $Y$, which is a $K$-equivariant endomorphism of $\left.S_{0}\right|_{Y}$. We assume that $\left.\Phi_{0}\right|_{Y}=\Phi_{0}^{Y} \otimes 1_{\mathfrak{p}}$, 
for a $K$-equivariant endomorphism $\Phi_{0}^{Y}$ of $S_{0}^{Y}$. (What follows remains true if $\Phi_{0}=\Phi_{0}^{Y} \otimes 1_{\mathfrak{p}}+1 \otimes \Phi_{0}^{\mathfrak{p}}$ for an $\operatorname{Ad}(K)$-invariant endomorphism of $S_{\mathfrak{p}}$, but this requires a small extra argument that we omit here.)

Consider the Dirac operator $D_{0}^{Y}:=c_{Y} \circ \nabla^{S_{0}^{Y}}$ on $\Gamma^{\infty}\left(S_{0}^{Y}\right)$. Form $D^{Y}$ from $D_{0}^{Y}$ as in (3.5) and $\Phi^{Y}$ from $\Phi_{0}^{Y}$ as in (3.6). Let $R(K)$ be the representation ring of $K$ and

$$
\mathrm{D}-\operatorname{Ind}_{K}^{G}: R(K) \rightarrow K_{*}\left(C^{*}(G)\right)
$$

be the Dirac induction map [4].

Corollary 7.4. The operator $D^{Y}+\Phi^{Y}$ is a $K$-equivariant Callias-type operator, and

$$
\operatorname{index}_{G}(D+\Phi)=\operatorname{D}-\operatorname{Ind}_{K}^{G}\left(\operatorname{index}_{K}\left(D^{Y}+\Phi^{Y}\right)\right) .
$$

Proof. Theorem 3.4 implies that $\operatorname{index}_{G}(D+\Phi)=\operatorname{index}_{G}\left(D^{S_{+}^{N}}\right)$. Write $Y^{N}:=Y \cap N$, so that $N=G \times{ }_{K} Y^{N}$. Then $Y^{N}$ is a compact manifold. Define $D^{S_{+}^{Y^{N}}}$ analogously to $D^{Y}$. The induction result for cocompact actions, Theorem, 4.5 in [19], Theorem 5.3 in [24] or Theorem 46 in [18], implies that

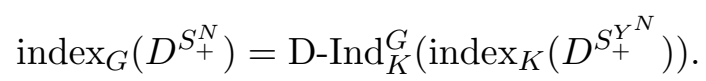

Another application of Theorem 3.4 , now with $G$ replaced by $K$, or Theorem 1.5 in [2] with a compact group action added, shows that $\operatorname{index}_{K}\left(D^{S_{+}^{Y^{N}}}\right)=$ $\operatorname{index}_{K}\left(D^{Y}+\Phi^{Y}\right)$.

7.5. Callias quantisation commutes with reduction. Theorem 3.11 in [16] is a quantisation commutes with reduction result for the equivariant index of $\mathrm{Spin}^{c}$-Callias-type operators. This result applies to reduction at the trivial representation of $G$; i.e. to an index defined in terms of $G$-invariant sections of $S$. Using Theorem 3.4, we can generalise this result to reduction at more general representations, or more precisely, at arbitrary generators of $K_{0}\left(C_{r}^{*}(G)\right)$. Furthermore, this result is 'exact' rather than asymptotic as Theorem 3.11 in [16, in the sense that one does not need to consider high powers of a line bundle.

In the setting of Subsection 3.2 , we now assume that $M$ is odd-dimensional, and that $S_{0}$ is the spinor bundle for a $G$-equivariant $\operatorname{Spin}^{c}$-structure. Let $D_{0}$ be the Spin ${ }^{c}$-Dirac operator on $\Gamma\left(S_{0}\right)$, defined by the Clifford connection corresponding to a connection $\nabla^{L}$ on the determinant line bundle $L$.

The $\operatorname{Spin}^{c}$-moment map associated to $\nabla^{L}$ is the map $\mu: M \rightarrow \mathfrak{g}^{*}$ such that for all $X \in \mathfrak{g}$,

$$
2 \pi i\langle\mu, X\rangle=\mathcal{L}_{X}-\nabla_{X^{M}}^{L}, \quad \in \operatorname{End}(L)=C^{\infty}(M, \mathbb{C}),
$$

where $\mathcal{L}_{X}$ denotes the Lie derivative with respect to $X$, and $X^{M}$ is the vector field induced by $X$. The reduced space at an element $\xi \in \mathfrak{g}^{*}$ is defined as $M_{\xi}:=\mu^{-1}(\xi) / G_{\xi}$, where $G_{\xi}$ is the stabiliser of $\xi$. This reduced space is noncompact in general, and may not be smooth. But the reduced space 
$N_{\xi}:=\left(\mu^{-1}(\xi) \cap N\right) / G_{\xi}$ is compact. It is not always a smooth manifold, but if it is, and $\xi \in \mathfrak{k}^{*}$, then we have an identification $N_{\xi} \cong Y_{\xi}^{N}:=\left(\mu^{-1}(\xi) \cap\right.$ $Y \cap N) / K_{\xi}$, with $Y$ and $Y^{N}$ as in Subsection 7.4, including Spin $^{c}$-structures. See Propositions 3.13 and 3.14 in [22]. Let $N^{+} \subset N$ be as in Corollary 7.3. Then we similarly have $N_{\xi}^{+} \cong Y_{\xi}^{N^{+}}:=\left(\mu^{-1}(\xi) \cap Y \cap N^{+}\right) / K_{\xi}$ in the smooth case, including Spin $^{c}$-structures.

There is a nontrivial way to define a $\operatorname{Spin}^{c}$-quantisation $Q^{\operatorname{Spin}^{c}}\left(Y_{\xi}^{N^{+}}\right) \in \mathbb{Z}$, even when $Y_{\xi}^{N^{+}}$is not smooth, described in detail in Section 5.1 of [31]. Motivated by the identification $N_{\xi}^{+} \cong Y_{\xi}^{N^{+}}$in the smooth case, we define $Q^{\operatorname{Spin}^{c}}\left(N_{\xi}^{+}\right):=Q^{\operatorname{Spin}^{c}}\left(Y_{\xi}^{N^{+}}\right)$for $\xi \in \mathfrak{k}^{*}$.

Let $T<K$ be a maximal torus, and fix a positive root system for $(K, T)$. Let $V \in \hat{K}$ have highest weight $\lambda \in i \mathfrak{t}^{*} \hookrightarrow \mathfrak{k}^{*} \hookrightarrow \mathfrak{g}^{*}$. (The first inclusion is defined by $\mathfrak{t}^{*} \cong\left(\mathfrak{k}^{*}\right)^{\mathrm{Ad}^{*}(T)}$, the second by the Cartan decomposition.) Following [31, 32, we call an element $\xi \in \mathfrak{k}^{*}$ an ancestor of $V$ if the coadjoint orbit $\operatorname{Ad}^{*}(K) \xi$ is admissible in the sense of [32, and its $K$-equivariant $\operatorname{Spin}^{c}$ quantisation is $V$. There exists a finite set $A(V)$ of ancestors representing all different such coadjoint orbits.

Let $C_{r}^{*}(G)$ be the reduced group $C^{*}$-algebra of $G$ and $\mathrm{D}$ - $\operatorname{Ind}_{K}^{G}$ the Dirac induction map (7.3). By the Connes-Kasparov conjecture, proved in [10, [26, 42], the abelian group $K_{*}\left(C_{r}^{*}(G)\right)$ is free, with generators D-Ind ${ }_{K}^{G}[V]$, where $V$ runs over $\hat{K}$.

Recall the definition of the Callias index of Spin ${ }^{c}$-Dirac operators (7.2).

Corollary 7.5 (Callias quantisation commutes with reduction). We have

$$
\operatorname{index}_{G}^{N^{+}}(D)=\bigoplus_{V \in \hat{K}} \sum_{\xi \in A(V)} Q^{\operatorname{Spin}^{c}}\left(N_{\xi}^{+}\right) \operatorname{D}-\operatorname{Ind}_{K}^{G}[V] \quad \in K_{*}\left(C_{r}^{*}(G)\right) .
$$

Proof. By Corollary 7.3 index $N_{G}^{N^{+}}(D)=\operatorname{index}_{G}\left(\left.D^{S_{0}}\right|_{N^{+}}\right)$, where now $D^{\left.S_{0}\right|_{N^{+}}}$ is a $\operatorname{Spin}^{c}$-Dirac operator on $N^{+}$. Theorem 4.6 in [22] implies that index $\operatorname{in}_{G}\left(\left.D^{S_{0}}\right|_{N^{+}}\right)$ equals

$$
\bigoplus_{V \in \hat{K}} \sum_{\xi \in A(V)} Q^{\operatorname{Spin}^{c}}\left(N_{\xi}^{+}\right) \operatorname{D}-\operatorname{Ind}_{K}^{G}[V]
$$

Remark 7.6. In cases where $M_{\xi}$ is smooth and $N_{\xi}$ is a hypersurface in $M_{\xi}$, which is a transversality condition between $N$ and $\mu^{-1}(\xi)$, one can use Theorem 1.5 in [2] (more precisely, its special case for $\mathrm{Spin}^{c}$-Dirac operators, which is the non-equivariant case of Corollary 7.3 to express the Spin ${ }^{c}$ quantisation $Q^{\operatorname{Spin}^{c}}\left(N_{\xi}^{+}\right)$as the index of a Callias-type operator on $M_{\xi}$.

\section{REFERENCES}

[1] Herbert Abels. Parallelizability of proper actions, global $K$-slices and maximal compact subgroups. Math. Ann., 212:1-19, 1974/75. 
[2] Nicolae Anghel. On the index of Callias-type operators. Geom. Funct. Anal., 3(5):431438, 1993.

[3] Michael Atiyah and Friedrich Hirzebruch. Spin-manifolds and group actions. In Essays on Topology and Related Topics (Mémoires dédiés à Georges de Rham), pages 18-28. Springer, New York, 1970.

[4] Paul Baum, Alain Connes, and Nigel Higson. Classifying space for proper actions and $K$-theory of group $C^{*}$-algebras. In $C^{*}$-algebras: 1943-1993 (San Antonio, TX, 1993), volume 167 of Contemp. Math., pages 240-291. American Mathematical Society, Providence, RI, 1994.

[5] Raoul Bott and Robert Seeley. Some remarks on the paper of Callias: "Axial anomalies and index theorems on open spaces" [Comm. Math. Phys. 62 (1978), no. 3, 213-234; MR 80h:58045a]. Comm. Math. Phys., 62(3):235-245, 1978.

[6] Maxim Braverman and Simone Cecchini. Callias-type operators in von Neumann algebras. J. Geom. Anal., 28(1):546-586, 2018.

[7] Ulrich Bunke. A $K$-theoretic relative index theorem and Callias-type Dirac operators. Math. Ann., 303(2):241-279, 1995.

[8] Constantine Callias. Axial anomalies and index theorems on open spaces. Comm. Math. Phys., 62(3):213-234, 1978.

[9] Simone Cecchini. Callias-type operators in $C^{*}$-algebras and positive scalar curvature on noncompact manifolds. ArXiv:1611.01800, 2016.

[10] Jérôme Chabert, Siegfried Echterhoff, and Ryszard Nest. The Connes-Kasparov conjecture for almost connected groups and for linear $p$-adic groups. Publ. Math. Inst. Hautes Études Sci., (97):239-278, 2003.

[11] Johannes Ebert. Elliptic regularity for Dirac operators on families of noncompact manifolds. ArXiv:1608.01699, 2018.

[12] Benyin Fu, Xianjin Wang, and Guoliang Yu. The equivariant coarse novikov conjecture and coarse embedding. ArXiv:1909.00529, 2019.

[13] Mikhael Gromov and H. Blaine Lawson, Jr. Positive scalar curvature and the Dirac operator on complete Riemannian manifolds. Inst. Hautes Études Sci. Publ. Math., (58):83-196 (1984), 1983.

[14] Victor Guillemin and Schlomo Sternberg. Geometric quantization and multiplicities of group representations. Invent. Math., 67(3):515-538, 1982.

[15] Hao Guo. Index of equivariant Callias-type operators and invariant metrics of positive scalar curvature. ArXiv:1803.05558, 2018.

[16] Hao Guo, Peter Hochs, and Varghese Mathai. Coarse equivariant callias index theory and quantisation. in preparation.

[17] Hao Guo, Peter Hochs, and Varghese Mathai. Equivariant Callias index theory via coarse geometry. ArXiv:1902.07391, 2019.

[18] Hao Guo, Varghese Mathai, and Hang Wang. Positive scalar curvature and Poincaré duality for proper actions. J. Noncommut. Geometry, 2019. DOI:10.4171/JNCG/321, ArXiv:1609.01404.

[19] Peter Hochs. Quantisation commutes with reduction at discrete series representations of semisimple groups. Adv. Math., 222(3):862-919, 2009.

[20] Peter Hochs. Quantisation of presymplectic manifolds, $K$-theory and group representations. Proc. Amer. Math. Soc., 143(6):2675-2692, 2015.

[21] Peter Hochs and Varghese Mathai. Spin-structures and proper group actions. Adv. Math., 292:1-10, 2016.

[22] Peter Hochs and Varghese Mathai. Quantising proper actions on $\operatorname{Spin}^{c}$-manifolds. Asian J. Math., 21(4):631-685, 2017.

[23] Peter Hochs, Yanli Song, and Xiang Tang. An index theorem for higher orbital integrals. in preparation, 2020.

[24] Peter Hochs and Hang Wang. A fixed point formula and Harish-Chandra's character formula. Proc. London Math. Soc., 00(3):1-32, 2017. 
[25] Dan Kucerovsky. A short proof of an index theorem. Proc. Amer. Math. Soc., 129(12):3729-3736, 2001.

[26] Vincent Lafforgue. $K$-théorie bivariante pour les algèbres de Banach et conjecture de Baum-Connes. Invent. Math., 149(1):1-95, 2002.

[27] H. Blaine Lawson, Jr. and Shing Tung Yau. Scalar curvature, non-abelian group actions, and the degree of symmetry of exotic spheres. Comment. Math. Helv., 49:232244, 1974.

[28] Eckhard Meinrenken. Symplectic surgery and the Spin ${ }^{c}$-Dirac operator. Adv. Math., 134(2):240-277, 1998.

[29] Alexandr S. Miščenko and Anatoliǐ T. Fomenko. The index of elliptic operators over $C^{*}$-algebras. Izv. Akad. Nauk SSSR Ser. Mat., 43(4):831-859, 967, 1979.

[30] Paul-Émile Paradan. Localization of the Riemann-Roch character. J. Funct. Anal., 187(2):442-509, 2001.

[31] Paul-Émile Paradan and Michèle Vergne. Equivariant Dirac operators and differentiable geometric invariant theory. Acta Math., 218(1):137-199, 2017.

[32] Paul-Émile Paradan and Michèle Vergne. Admissible coadjoint orbits for compact Lie groups. Transform. Groups, 23(3):875-892, 2018.

[33] Markus Pflaum, Hessel Posthuma, and Xiang Tang. The transverse index theorem for proper cocompact actions of Lie groupoids. J. Differential Geom., 99(3):443-472, 2015.

[34] Paolo Piazza and Hessel Posthuma. Higher genera for proper actions of Lie groups. ArXiv:1801.06676, 2018.

[35] Jonathan Rosenberg. $C^{*}$-algebras, positive scalar curvature, and the Novikov conjecture. Inst. Hautes Études Sci. Publ. Math., (58):197-212 (1984), 1983.

[36] Jonathan Rosenberg. $C^{*}$-algebras, positive scalar curvature and the Novikov conjecture. II. In Geometric methods in operator algebras (Kyoto, 1983), volume 123 of Pitman Res. Notes Math. Ser., pages 341-374. Longman Sci. Tech., Harlow, 1986.

[37] Jonathan Rosenberg. $C^{*}$-algebras, positive scalar curvature, and the Novikov conjecture. III. Topology, 25(3):319-336, 1986.

[38] Thomas Schick and Mostafa Esfahani Zadeh. Large scale index of multi-partitioned manifolds. J. Noncommut. Geom., 12(2):439-456, 2018.

[39] Youliang Tian and Weiping Zhang. An analytic proof of the geometric quantization conjecture of Guillemin-Sternberg. Invent. Math., 132(2):229-259, 1998.

[40] Bai-Ling Wang and Hang Wang. Localized index and $L^{2}$-Lefschetz fixed-point formula for orbifolds. J. Differential Geom., 102(2):285-349, 2016.

[41] Hang Wang. $L^{2}$-index formula for proper cocompact group actions. J. Noncommut. Geom., 8(2):393-432, 2014.

[42] Antony Wassermann. Une démonstration de la conjecture de Connes-Kasparov pour les groupes de Lie linéaires connexes réductifs. C. R. Acad. Sci. Paris Sér. I Math., 304(18):559-562, 1987.

TeXas A\&M University

E-mail address: haoguo@math.tamu.edu

University of Adelaide

E-mail address: peter.hochs@adelaide.edu.au

UNIVERSITY OF ADELAIDE

E-mail address: mathai.varghese@adelaide.edu.au 